\title{
2. Über den Zusammenhang der dielektrischen Effektverluste von Kondensatoren mit den Anomalien der Ladung und der Leitung; von Franะ Tank.
}

(Zürcher Dissertation.)

\section{Finleitung.}

Seit Siemens ${ }^{1}$ ) 1864 nachwies, daB in Kondensatoren, die einem elektrischen Wechselfeld ausgesetzt sind, Energieverluste auftreten, die sich in einer Erwärmung des Dielektrikums äußern, sind diese Verluste Gegenstand zahlreicher Untersuchungen ${ }^{2}$ ) gewesen. Sie beanspruchen heute insbesondere das praktische Irteresse der Technik, da sie sich bei den hohen Spannungen der Starkstromtechnik ${ }^{8}$ ) and den hohen Frequenzen der Hochfrequenztechnik ") deutlich fühlbar machen.

Man war früher geneigt, in der "Siemenswärme" ein direktes Analogon zur magnetischen Hysteresiswärme zu sehen.5) Nach anderen Auffassungen wurde sie als Joulesche Wärme interpretiert, bedingt durch eine gewisse Leitfähigkeit des Dielektrikums.") Spätere Untersuchungen suchten sie mit den allgemeinen dielektrischen Nachwirkungserscheinungen, die auch Nachladung, Rückstand usw. bedingen, in Zusammenhang zu bringen. E. v. Schweidler vergleicht in seinen „Studien über die Anomalien im Verhalten der

1) W. Siemens, Pogg. Ann. 125. p. 137. 1864.

2) Literatur vor 1907 vgl. das Literaturverzeichnis in E. v. Sch weidler, Ann. d. Phys. 24. p. 767. 1907.

3) Vgl. z. B. M. Höchs täd ter, Elektroteohn. Zeitschr. p. 467. 1910.

4) Vgl. z. B. W. Hahnemann u. L. Adelmann, Elektrotechn. Zeitschr. p. 988 u. 1010, 1807.

5) Vgl. z. B. A. Kleiner, Wied. Ann. 50. p. 138.1893.

B) G. Benischke, Wiener Sitzungsber. 1893; Porter u. Morris, Proc. Roy. Soc. 57. 1895. 
Dielektrika "1) diese verschiedenen Frklärungsmöglichkeiten ausführlich. Ausgehend von den statischen dielektrischen Nachwirkungserscheinungen gelangt er durch weitere Fassung der Ansätze von H. Pellat ${ }^{2}$ ) und J. Hopkinson ${ }^{3}$ ) zu einer Theorie der Dielektriken, in der sich alle Erscheinungen anomalen Verhaltens 2usammenfassen lassen. Diese Theorie ist von K. W. Wagner ${ }^{4}$ ) in engster Anlehnung an die Wiechertsche Theorie der els,stischen Nachwirkung ${ }^{5}$ ) ausgebaut worden und vermag die experimentellen Ergebnisse der verschiedenen Beobachter ${ }^{6}$ ) befriedigend darzustellen.

Es läßt sich stuf rein experimenteller Grundlage leicht zeigen, dab ein sogenanntes unvcllkommenes Dielektikum, als welches stets ein mit Nachwirkung behaftetes Dielektrikum bezeichnet werden soll, im elektrischen Wechselfelde Fnergieverluste aufweisen muß, deren Berechnung bei Kenntnis einer gewissen Nachwirkungsfunktion möglich ist. Die Wagnersche Theorie gibt für diese einen Ansatz, der von Wiechert übernornmen ist. Man kann aber auch, nach einem Vorschlage $E_{1}$. v. Schweidlers, sio experimentell aus den Anomalien der: Ladung, $d . h$. dem zeitlichen Verlauf von Nachladung und Rückstandsbildung, bestimmeı. Da die meisten Dielektriken eine gewisse Leitfähigkeit besitzen, werden zu diesen Verlusten noch Verluste durch Leitung hinzutreten. Diese befolgt im allgemeinen das Ohmsche Gesetz nicht und es wird einer besonderen Untersuchung ihrer Eigenschaften bedürfen, um ihren Anteil am Gesamtverlust festzustellen. Drittens läBt sich nicht von vornherein entschciden, ob im Wechselfelde nicht noch zusätzliche Energieverluste vorkommen, die sich weder aus der Betrachtung der Nachwirkung noch der Lreitung ergeben.

Es soll daber der Zweck der vorliegenden Arbeit sein.

1) E. v. Schweidler, Ann. d. Phys. 24. p. 767. 1907.

2) H. Pellat, Compt. rend. 128. p. 1312. 1899; Ann. chim. phys. (7) 18. p. 150 1899; Journ. de Phys. (3) 9. p. 313. 1900.

3) J. Ho pkinson, Phil. Trans. 166. p. 489. 1876; 167. p. 599. 1877/78; Phil. Mag. (5) 2. p. 314. 1876.

4) K. W. Wagner, Ann. d. Phys. 40. p. 817. 1913; auch Arch. f. Elektroteohn. 2. p. 372. 1914; 3. p. 67. 1914.

5) E. Wiechert, Inaug.-Diss. Königsberg 1889; Wied. Ann. 50. p. 548. 1893.

6) Vgl. K. W. Wagner, l, c. 
auf experimentellem Wege durch Untersuchung der Anomalien der Ladung und der Leitung, die Beiträge ron Nachwirkung und Leitung zum. Gesamtverlust gesondert zu bestimmen. Ferner soll letzterer direkt gemessen werden. Ein Vergleich wird dann zeigen, wie sich die einzelnen Anteile zu diesem verbalten und ob Schlüsse auf seine Natur möglich sind.

\section{Theoretische Grundlagen.}

\section{Die Anomalien der Ladung.}

Wird ein Kondensator durch einen induktionsfreien Widerstand mit einer Stromquelle von der konstanten elektromotorischen Kraft $E$ verbunden, so erfolgt zunächst eine "normale Ladung", bei der die Stromstärke, bei einem endlichen Werte beginnend, exponentiell auf Null abklingt. Besitzt das Dielektrikum nicht ideale Eigenschaften, ist es leitend und rückstandbildend, so üborlagert sich diesem ",normalen Ladungisstrom" ein "normaler Leitungsstrom", bestimmt durch die Leitfähigkeit und die geometrischen $\mathrm{Ab}$ mossungen des Kondensators, und weiter ein ,enomaler Ladungsstrom" oder "Nachladungsstrom". Ähnlich wie der normale Ladungsstrom nähert er sich mit der Zeit dem Werte Null, jedoch viel langsamer, so dab er meist viele Minuten nach erfolgter normaler Ladung noch nachweisbar ist.

Für den Nachladungsstrom wurden experimentell folgende GesetzmäBigkeiten gefunden ${ }^{1}$ ):

a) Er ist proportional der Kapazität $C$ des Kondensators und proportional der Potentialdifferenz $E$ der Belegungen. Er muB sich also ausdrücken lassen durch eine Gleichung

$$
i=E \cdot C \cdot \varphi(t),
$$

wo $\varphi(t)$ eine Funktion der Zeit ist, die außerdem für das Dielektrikum charakteristische Konstanten enthält. Diese Konstanten sind als Konstanten der dielektrischen Nachwirkung anzusehen.

b) Er befolgt das Superpositionsprinzip, d. h. ändert man zur Zeit ( $\delta$ ) die Spannung $E$ um den Betrag $E$, so ergibt 1888.

1) J. Hopkinson, l. c.; J. Curie, Ann. chim. phys. (6) 18. p. 203. 
sich ein Strom, der aus der Ùbereinanderlagerung des ursprünglichen Stromes

$$
i=E \cdot C \cdot \varphi(t)
$$

und des der Änderung $\Delta E$ entsprechenden Stromes

$$
\boldsymbol{\Delta} i=\Delta E \cdot C \cdot \varphi(t-\delta)
$$

resultiert. Ist die Spannungsdifferenz eine stetige, aber sonst keliebige Funktion von

$$
E=E(\delta),
$$

so verallgemeinert sich der obige Ausdruck zu

$$
i=C \int_{-\infty}^{t} \frac{d E(\delta)}{d \delta} \varphi(t-\delta) d \delta,
$$

oder wenn man $t-\delta=u$ setzt:

$$
i=-C \int_{0}^{\infty} \frac{d E(t-u)}{d u} \varphi(u) d u .
$$

Es ist $u$ die Integre,tionsvariable und $t$ als ein fixierter Zritmoment zu denken.

Aus dieser Formel läßt sich $u$, a. berechnen, daß der Strom, der nach vorausgegangener sehr langer Ladung der „normalen“ Entladung des Kondensators folgt, der sogenannte "Rückstandsstrom" $i$ ' dem Nachladungsstrom $i$ entgegengesetzt gleich ist:

$$
i=-i^{\prime} \text {, }
$$

welche Beziehung u. a. durch E. v. Schweidler ${ }^{1}$ ) und besonders genau durch $\mathrm{K}$. W. Wagner ${ }^{2}$ ) geprüft und bestätigt worden ist. Man kann sich daher vorstellen, daß die durch den Nachladungsstrom zugeführte Elektrizitätsmenge im Kondensator aufgespeichert wird und nachher im Rückstandsstrom wieder frei wird. Die Rückstandsladung bei der Ladezeit $T$ ist dann

$$
R=\int_{0}^{T} i d t
$$

1) E. จ. Sohweidler, l. c. p. 753.

2) K. W. Wagner, l. c. p. 844. 
oder insbesondere bei Ladung durch eine konstante elektromotorische Kraft $E$ :

$$
R=E C \int_{0}^{T} \varphi(t) d t
$$

Ihr entspricht eine scheinbare Kapazitätsvergrößerung vom Betrage:

$$
\Delta C=\frac{R}{E} .
$$

Da ein Kondensator mit unvollkommenem Dielektrikum stets eine von der Ladezeit und der Vorgeschichte abhängige Kapazität aufweisen wird, ist es zweckmäBig, die von Nachwirkung freie, $d . h$. auf sehr kurze Ladezeit reduzierte Kapazität einzuführen. Sie sei im folgenden mit $C_{0}$ bezeichnet; H. L. (urtis $^{1}$ ) nennt sie die geometrische Kapazität.

\section{Die Darstellung des Nachladungastromes durch}

die v. Bohweidler-Wagnersche Theorie der dielektrischen Nachwirkung.

Der Verlauf.des Nachladungsstromes bei Ladung eines Kondensators durch eine konstante elektromotorische Kraft wurde von älteren Beobachtern ${ }^{2}$ ) durch direkte galvanometrische Messung übereinstimmend gefunden an:

$$
i=E C_{0} \beta t^{-n} \quad 0<n<1 \text {; }
$$

daher die Funktion $\varphi(t)$, die das anumale Verhalten des Dielektrikums charakterisiert:

$$
\varphi(t)=\beta t^{-n}
$$

mit zwei Materialkonstanten $\beta$ und $n$, wobei $n$ stets Werte zwischen Null und Eins besaß. Schon E. v. Schweidler ${ }^{3}$ ) weist darauf hin, daß diese Formel nur als gute Näherung innerhalb eines beschränkten Gültigkeitsbereiches aufzufassen ist. Sowohl für kleine als für sehr große Zeiten ergeben sich physikalisch unwahrscheinliche Konsequenzen: für $t=0$ wird der Nachladungs- bzw. Rückladungsstrom, für $t=\infty$ die Rückstandsladung unendlich groß.

1) H. L. Curtis, Bull. of Stand. Washington 6. p. 471. 1910.

2) Vgl. z. B. R. Kohlrausch, Pogg. Ann. 91. p. 56. 1854; W. Giese, Wied. Ann. 8. p. 161. 1880; J. Hopkinson, l. c.; J. Curie, 1. c.

3) E. v. Sohweidler, 1. c. p. 715 u. 745. 
Diese Schwierigkeiten vermeidet der Ansatz von H. Pellat ${ }^{1}$ ), der dem Nachladungsstrom exponentiellen Verlauf zuschreibt. Pellat setzt:

$$
\begin{gathered}
\varphi(t)=\beta e^{-\frac{t}{T}}=\frac{k}{T} e^{-\frac{t}{T}}, \\
i=E C_{0} \frac{k}{T} e^{-\frac{t}{T}} .
\end{gathered}
$$

Er faßt den Nachladungsstrom als einen Verschiebungsstrom in Dielektrikum auf, herrührend von einer Verschiebung

$$
\vartheta^{\prime}=-\vartheta_{0} k e^{-\frac{t}{T}}
$$

$\vartheta_{0}$ soll die Verschiebung sein, die bei gleichem elektrischen Feld der Kapazität $C_{0}$ zukäme. K. W. Wagner nennt sie Ausgleichsverschiebung, E. v. Schweidler ${ }^{2}$ ) spricht von viskoser Verschiebung. Sie ist die Differenz des momentanen Wertes und des nach sehr langer Zeit erreichten Endwertes der Verschiebung $\vartheta$ :

$$
\vartheta^{\prime}=\vartheta-\vartheta_{\infty}
$$

Da diese Forrnulierung der Erfahrung nicht genügt, erweiterte sie E. v. Schweidler dadurch, daß er eine Zahl von Ausgleichsverschiebungen als unabhängig nebeneinander bestehend annahm, also den einfachen Pellatschen Ausdruck durch eine Summe ersetzte:

$$
\begin{gathered}
\vartheta^{\prime}=-\vartheta_{0} \sum_{n} k_{n} e^{-\frac{t}{T_{n}}}=-\vartheta_{0} \psi(t) ; \\
\sum_{n} k_{n} e^{-\frac{t}{T_{n}}}=\psi(t) .
\end{gathered}
$$

Diese Gleichung läßt eine molekularphysikalische Deutung ru, wenn man annimmt, $\mathrm{daB}$ in einem Nachwirkung zeigenden Dielektrikum Elektronen, Ionen oder Ionenkomplexe vorhanden sind, die nicht, wie die "Polarisationselektronen", Eigenschwingungen von bestimmter Dauer und Dämpfung ausführen, sondern deren Bewegung aperiodisch verzögert ist. Unter dem EinfluB eines konstanten elektrischen Feldes nähern sie sich ihrer neuen Gleichgewichtslage mit einer Geschwindig-

1) H. Pellat, l. c.

2) E. v. Schweidler, l. c. p. 742 . 
keit, die proportional ist zu $e^{-\frac{t}{T}}$. Die Annahme verschiedener Zeitkonstanten $T_{n}$ und verschiedener Verhältniskonstanten $k_{n}$ kommt so der Annahme verschiedener Gattungen dieser Ionen gleich.

Es ist nahe gelegt, diese Betrachtung auf sehr viele Ionen mit ganz verschiedenen $T_{n}$ und $k_{n}$ auszudebnen, also obige Summe in ein Integral übergehen zu lassen:

$$
\vartheta^{\prime}=-\vartheta_{0} \int_{0}^{\infty} k(T) e^{-\frac{t}{T}} d t ; \quad \psi(t)=\int_{0}^{\infty} k(T) e^{-\frac{t}{T}} d t
$$

und den einzelnen Gliedern ein' gewisses Wahrscheinlichkeitsgewicht beizulegen. Dieser Gedanke, durch E. v. Schweid ler vorbereitet ${ }^{1}$ ), ist von $K$. W. Wagner ${ }^{2}$ ) durchgeführt worden. Der unmittelbare AnschluB, der sich dabei an Wiecherts Theorie ${ }^{3}$ ) der elastischen Nachwirkung ergibt, läBt die Möglichkeit vermuten, die dielektrische Nachwirkung einem Gebiet der allgemeinen Nachwirkungserscheinungen einzuordnen.

Bezüglich der näberen Ausführung auf die zitierten wichtigen Arbeiten verweisend, sei hier kurz zusammengefaBt:

$k(T)$ ist, wie dies schon aus der Schreibweise hervorgeht, als Funktion des jeweiligen Wertes $T$ der Zeitkonstanten aufzufassen. Man kann sie die „Verteilungsfunktion“ der Zeitkonstanten nennen. Unter einfachen Voraussetzungen gibt die Wahrscheinlichkeitsrechnung dafür den Ausdruck

$$
k(T) d T=\frac{k b}{\sqrt{\pi}} e^{-b^{2} z^{2}} d z,
$$

wobei :

$$
z=\log \operatorname{nat} \frac{T}{T_{0}} .
$$

Damit ist gesagt, daB die Logarithmen der Zeitkonstanten sich um einen wahrscheinlichsten Wert log nat $T_{0}$ gruppieren sollen, und zwar um so dichter, je größer die Konstante $b$ ist. Gleichungen (3) und $\left(\mathbf{3}^{\prime}\right)$ gehen dann über in

1) E. v. Sohweidler, l. c. p. 748.

2) K. W. Wagner, l. o.

3) E. Wiechert, l. c. 


$$
\begin{aligned}
& \vartheta^{\prime}=-\vartheta_{0} \frac{k b}{\sqrt{\pi}} \int_{-\infty}^{+\infty} e^{-b^{z} z^{2}-\frac{t}{T} e^{-z}} d z \\
& \psi(t)=\frac{k b}{\sqrt{\pi}} \int_{-\infty}^{+\infty} e^{-b^{2} z^{2}-\frac{t}{T} e^{-z}} d z
\end{aligned}
$$

und für den Nachladestrom erhält man

$$
\begin{aligned}
& i=-E C_{0} \frac{d \psi}{d t}, \\
& i=-E C_{0} \frac{k b}{T_{0} \sqrt{\pi}} \int_{-\infty}^{+\infty} e^{-b^{4} z^{2}-z-\frac{t}{T_{0}} e^{-z}} d z .
\end{aligned}
$$

Dieses Integral läßt sich für kleine Werte von $b$ durch den bequemeren Näherungsausdruck ersetzen:

$$
i=-E C_{0} \frac{k b}{T_{0} \sqrt{\pi}} \cdot \frac{T_{0}}{t} e^{-\left(b \log \text { nat } \frac{t}{T_{0}}\right)^{2},}
$$

woraus durch logarithmieren unmittelbar hervorgeht

$$
\log i=\text { konst }-\log \frac{t}{T_{0}}-\frac{b^{2}}{\log e}\left(\log \frac{t}{T_{0}}\right)^{2}
$$

Im logarithmischen Koordinatensystem stellt diese Gleichung eine Parabel dar. Begrenzte Stücke von ibr können in der Umgebung des Zeitpunktes $t=\tau$ durch eine Gerade ersetzi werden, deren Form dann lautet:

$$
\log i=\text { konst }-\left(1+2 b^{2} \log \text { nat } \frac{x}{T_{0}}\right) \log t .
$$

Dies ergibt einen Stromverlauf, der identisch wird mit dem empirisch bestimmten

oder

$$
i=B t^{-n}
$$

$$
\log i=\text { konst }-n \log t \quad 0<n<1 .
$$

Dadurch ist letztere Formel als bloße empirische Näherungsformel erklärt. Der relativ große Gültigkeitsbereich beweist aber, daß bei den meisten Dielektriken kleines $b$, also ein breites Gehiet weit auseinanderliegender Zeitkonstanten $T$ anzunehmen ist. Der Nachweis, daß dieser Gültigkeitsbereich sich auch auf sehr kleine Zejten ausdehnt, wird für uns be- 
sondere Bedeutung haben, weil dann eine bequeme genäherte Berechnung der Finergieverluste im elektrischen Wechselfeld möglich sein wird.

\section{Die Berechnung der dielektrischen Wechselstromverluste aus dem Verlane des Nachladungestromes.}

Während der Strom eines idealen Kondensators bei sinusförmiger Spannung $E=E_{0} \sin (\omega t)$ gegeben ist durch

$$
J_{0}=\omega C_{0} E_{0} \cos (\omega t)
$$

verursachen Effektverluste eine Phasenverschiebung und eine scheinbare Änderung der Kapazität, so da $B$ der Strom in der Form geschriehen werden mu $B$ :

$$
J=\omega C_{0} E_{0}\{a \cos \omega+b \sin \omega t\} .
$$

Er läBt sich also, entsprechend der bekannten Darstellung eines Verluste aufweisenden Kondensators durch eine ideale Kapazität mit parallel geschaltetem Widerstand, in zwei Komponenten zerlegen: aus der einen Komponente läßt sich die ,scheinbare Kapazität" $a C_{0}$, aus der anderen der ,scheinbare Leitwert" $\omega C_{0} b$ ersehen. Für den Fffektverlust folgt:

$$
Q=\frac{1}{2} \omega C_{0} E_{0}^{2} b \text {. }
$$

Es ist nun ohne weiteres anschaulich, daß bei einem Kondensator, der dielektrische Nachwirkung zeigt, bei dem also die Ladung immer erst allmählich ihren vollen Betrag erreicht, ein Zurückbleiben der I.adurg hinter der Spannung und somit eine Phasenverschiebung auftreten muB. Dies bedingt das Zustandekommen einer Art von „Hysteresisschleifen", über die in der Tat zahlreiche Beobachtungen vorliegen. ${ }^{1}$ ) Daß diese Schleifen bei Glas und imprägnierten Papieren besonders ausgeprägt sind (C. Lomsché, H. Nuss baumer), wird durch unsere noch mitzuteilenden Versuchsresultate eine Erklärung und Bestätigung finden. Die Be-

1) Janet, Journ. de phys. (3) 2. 1893; Beaulard, Ann. Univ. Grenoble 18. 1900; Journ. de phys. (3) 9. 1900; Eisler, Elektrotechn. Zeitschr. Juni 1895; Mercanton, Thèse Lausanne 1902; C. Lomsch6́, Diss. Zürich 1903; H. Nussbaumer, Diss. Zürich 1907; M. Höch städter, Elektrotechn. Zeitachr., Mai und Juni 1910. 
rechnung der Stjomstärke und des Effelitverlustes kann auf Grund der allgemeinen Gleichung $\left(\mathbf{2}^{\prime}\right)$ geschehen. Sie lautete:

$$
i=-C_{0} \int_{0}^{\infty} \frac{d E(t-u)}{d u} \varphi(u) d u .
$$

Ist $E=E_{0} \sin \omega t$, so ist $E(t-u)=E_{0} \sin [\omega(t-u)]$, und es wird:

$$
i=\omega C_{0} E_{0}\{A \cos \omega t+B \sin \omega t\}
$$

wobei

$$
\left\{\begin{array}{l}
\left.A=\int_{0}^{\infty} \varphi(u) \cos (a) u\right) d u, \\
B=\int_{0}^{\infty} \varphi(u) \sin (\omega u) d u .
\end{array}\right.
$$

Der Gesamtstrom im Kondensator setzt sich daher zusammen aus :

a) dem ,norralen Strom" $J_{0}=\omega C_{0} E_{0} \cos \omega t$;

b) dem Nachwirkungsstrom $i$ [Gleichung (8)];

c) dem „normalen Leitungsstrom", der, konstante Leitfähigkeit vorausgesetzt, einem "Gleichstromwiderstand" $W$ entspricht und gesschrieben werden kann:

$$
j=\frac{E}{W} \sin \omega t
$$

So ergibt sich

$$
J=J_{0}+i+j .
$$

(10) $J=\omega C_{0} E_{0}\left\{(1+A) \cos \omega t+\left(\frac{1}{\omega C_{0} W}+B\right) \sin \omega t\right\}$

und für die scheinbare Kapazität:

$$
C=C_{0}(1+A) \text {, }
$$

und für den Effeltverlust:

$$
Q=\frac{1}{2} \omega C_{0} E_{0}^{2}\left\{\frac{1}{\omega C_{0} W}+B\right\} .
$$

Die Integrale $A$ und $B$, die die Kapazitätsänderung sowie den Verlust in Abhängigkeit von der Frequenz des Wechselstromes bestimmen und den Zusammenhang mit den dielektrischen Nachwirknngserscheinungen statischer Natur quantitativ vermitteln, lassen sich auswerten, sobald die Funktion 
$\varphi(u)$ bekannt ist. Für $\varphi(u)=\beta u^{-n}, 0<n<1$, hat E. v. Schweidler ${ }^{1}$ ) diese Rechnung durchgeführt. Man erhält:

$$
\left\{\begin{array}{l}
A=(\omega)^{n-1} \cdot \beta \cdot \Gamma(1-n) \cos \left\{\frac{(1-n) \pi}{2}\right\} \\
B=(\omega)^{n-1} \cdot \beta \cdot \frac{\pi}{2 \Gamma(n) \cos \frac{(1-n) \pi}{2}}
\end{array}\right.
$$

$\Gamma$ ist das bekannte Eulersche Integral. Ist noch der Isolierwiderstand ermittelt, so ist Gleichung (12) die Beziehung, nach der der Anteil der dielektrischen Nachwirkung and der Leitfähigkeit an den Wechselstromeffekteverlusten berechnet werden kann. Durch Vergleich mit den direkt gemessenen Verlusten wird sich die Größe dieses Anteiles feststellen lassen.

Man kann nun empirisch, ohne weitere Voraussetzungen, die Größen $A$ und $B$ erhalten, wenn es gelingt, den Verlauf des Nachladungsstromes für sehr kleine Zeiten, die mit der Periodendauer der Wechselströme vergleichbar sind, aufzunehmen.

Denn für den Nachladungsstrom gilt ja nach Gleichung (1):

$$
i=E C_{0} \varphi(t) ;
$$

er enthält also unmittelbar die Funktion $\varphi$. Von dieser weiß man daher, daß sie für kleine Argumentwerte sehr groß wird und mit wachsendem Argument rasch abnimmt und sich sehr kleinen Werten nähert. Ist $\varphi(t)$ für kleine Argumente experimentell festgelegt und durch eine Näherungsgleichung dargestellt, so werden sich durch Einsetzen in die Ausdrücke (9) $A$ und $B$ näherungsweise bestimmen lassen. Das Beobachtungsintervall ist dem verlangten Grad.der Genauigkeit entsprechend zu wäblen; das Verfahren wird nur für solche Dielektrika zum Ziele führen können, deren Nachladungsstrom bei wirklich großen Werton beginnt und rasch abfällt, also für solche, die in kurzer Zeit beträchtliche Rückstandsladung bilden.

Die Einsetzung des Wertes

$$
\varphi(u)=\beta u^{-n} ; \quad 0<n<1,
$$

1) E. v. Schweidler, 1. c. p. 723. 
wie er aus dem Verlauf des Nachladungsstromes

$$
i=E C_{0} \beta t^{-n} ; \quad 0<n<1
$$

nach den experimentellen Bestimmungen von Kohlrausch, Giese, Hopkinson, Curie, v. Schweidler u. a. folgt, ist daher einigermaßen bedenklich, weil diese Messungen sich in einem Zeitintervall von $t=10$ Sek. bis einigen Stunden bewegen, über kleine Zeiten also nichts aussagen. Der einzige Versuch, der nir bekannt ist, ans dem Verlauf des Nachladungsstromes die dielektrischen Verluste $v u$ bestimmen und mit direkten Weshselstrommessungen $\mathrm{zu}$ vergleichen, ist die Arbeit von $H$. Jordan. ${ }^{\text {) }}$

$H$. Jordan gelang es, durch eine verbesserte Methode mit dem ballistischen Galvanometer die Untersuchung auf $0,13<t<1,3$ Sek. auszudehnen. Fr findet, daß dia Kurven des Nachladungsstromes ungefähr logarithmisch verlaufen, daB sich aber ,auch nicht schälzungsweise ein Anfangswert für $l=0$ angeben läft, ebensowenig ein endlicher Grenzwert für $t=\infty$. Hierdurch sind einerseits die Extrapolationen für die kleinen Zeiten von der Größenordnung der Periode der Wechselströme unsicher; andererseits ist keine Andeutung vorhanden, $\mathrm{da} B$ ein endlicher Grenzwert entsprechend der normalen Leitfähigkeit erreicht wird". Doch kommt er zu dem Resultat, „daB an Hand der Theorie E. v. Schweidlers für ein und dasselbe Dielektrikum der Zusammenhang zwischen den so sehr verschiedenen wirksamen Leitwerten (oder Isolationswiderständen) bei Messungen mit Wechselstrom der praktisch in Frage kommenden Frequenzen und mit ,Gleichstrom' gezeigt ist, und daß es möglich ist, aus diesen wenigstens näherungsweise zu extrapolieren auf die Wechselstromleitwerte für nicht zu große Frequenzen ".

Ich stellte mir daher die Aufgabe, diesen Gedankengang nit anderen Methoden zu verfolgen, um so einen Einblick in die Natur der dielektrischen Effektverluste zu erhulten. Ich bestimmte mit dem Helmholtzschen Pendel den Verlauf des Nachladungsstromes für möglichst kleine Zeiten und maß den Isolierwiderstand des Dielektrikums gegen Gleichstrom. Nach dem angedeuteten Verfahren orgab dann Gleichung (12) den notwendigen Arteil von dielektrischer Nach-

1) H. Jordan, Verh. d. Deutsch. Phys. Ges. 14. p. 451.1912. 
wirkung und Leitfähigkeit an den Wechselstromverlusten. Diese wurden mit Hilfe eines Elektrodynamometers bei einer der verwendeten Gleichstromspannung gleichen Effektivspannung gemessen. Man erhielt so auf vollständig verschiedenen Wegen zwei Resultate für den Effektverlust, die zu vergleichen waren.

II. Beschreibung der Versuche.

1. Die Bestimmung des Nachladungestromes mit dem Helmholtzgchen Pendel.

a) Das Helmboltzsohe Pendel.

Wie am Schlub des vorigen Abschnittes ausgeführt, ist, es entscheidend, den Verlauf des Na.chladungsstromes für kleine Zeiten zu kennen. Während für diesen Zweck die galvanometrischen Methoden versagen, erweist sich die Verwendung des Helmholtzschen Pendelunterbrechers als geeignet.

Ich konnte ein Pendel in einer von Prof. Dr. A. Kleiner konstruierten Form benutzen. Da das Instrunient wiederholt beschrieben worden ist ${ }^{1}$ ), erübrigt wohl eine nähere Darstellung. Das eigentliche Pendelstück besitzt die Form eines Kreisquadranten, durch dessen rechtwinklige Ecke die Drehachse geht, und längs dessen Bogen auf beiden Seiten Schlagstifte verschiebbar sind; ihre Stellung kann an einer Gradteilung abgelesen werden. Es wird so fixiert, daß sein Schwerpunkt mit der Drehachse in einer Horizontalebene liegt; beim Aufheben der Fixierung, was durch Auslösung vermittelst einer Schnur vom Platze des Beobachters aus geschehen kann, fällt es, und die Stifte schlagen zwei Kontakthebel nacheinander weg. Von letzteren ist die Lage des einen fest; der andere kann auf einem Schlitten mit einer Mikrometerschraube verschoben werden. Das Keitintervall, das zwischen dem Wegschlagen des ersten und des zweiten Kontakthebels liegt, kann also auf zweierlei Weise reguliert werden: für größere Intervalle durch Finstellung der Schlagstifte, für kleinere Intervalle mit der Mikrometerschraube.

Durch die Bestimmung der Ladekurve eines Kondensators, dem ein induktionsfreier Widerstand und eine konstante

1) Vgl. z. B. G. Eichhorn, Inaug.-Diss. Zürich 1901. 
elektromotorische Kraft vorgeschaltet sind, wurde die Eichung des Pendels vorgenommen. ${ }^{1}$ ) Gute Präzisionskondensatoren laden sich sehr genau nach der theoretisch verlangten Formel auf. Es ist empfehlenswert, nur den Beginn der Ladung zur Messung zu benutzen; die Ladung verläuft dann merklich linear und kann für alle Einstellungen gleich genau bestimmt werden; sollte der Kondensator nicht nachwirkungsfrei sein, so überwiegt da,nn auch der ,normale Ladungsstrom" den „Nachladungsstrom“ noch. vollständig.

Die Mittel aus je sechs MeBreihen, ausgeführt mit verschiedenen Kapazitäten, Widerständen und elektromotorischen Kräften ergaben :

a) Mikrometerschraube:

1 Trommelteil $=0,00500 \mathrm{~mm}=(1,641 \pm 0,005) \cdot 10^{-6}$ Sek.

b) Pendelquadrant:

Winkeldistanz der Schlagstifte

$1^{0}$
2
10
20
30
40
60
90

Zeitwert $t$
0,00233 Sek.
0,00467
0,02347
0,04695
0,07045
0,08252
0,1440
0,2265

Zeitwert $t$

0,00467

0,02347

0,04695

0,08252

0,2265

Die Meßgenauigkeit beträgt etwa $1 / 3$ bis $1 / 2$ Proz.

\section{b) Die Meßanordnung.}

Die Versuchsanordnung zur Bestimımung des Nachladestromes ist in beistehender Zeichnung skizziert, der auch eine Tabelle der Bezeichnungen und Maße beigegeben ist.

Der Grundgedanke ist der, $\mathrm{da} B$ an den Versuchskondensator $C$ die Spannung $E$ verschieden lang dauernde Zeit angelegt wird. Der Widerstand $W$ wird so bemessen, daB die „normale Ladungszeit" des Kondensators gegen diese Zeit meist klein ist, so daB man den Kondensator während des Versuches als unter konstanter Spannung stehend betrachten kann. Man mißt die Elektrizitätsmenge, die durch die $\mathrm{Zu}$ leitung hindurchgeströmt ist, dadurch, daß man sie auf einen

1) Vgl.'z. B. C. Lomsché, Inaug.-Diss. Zürich 1903. 
Hilfskondensator $G$ großer Kapazität ansammelt und am Quadrantelektrometer dessen Potential ermittelt.

Schaltungsschema I.

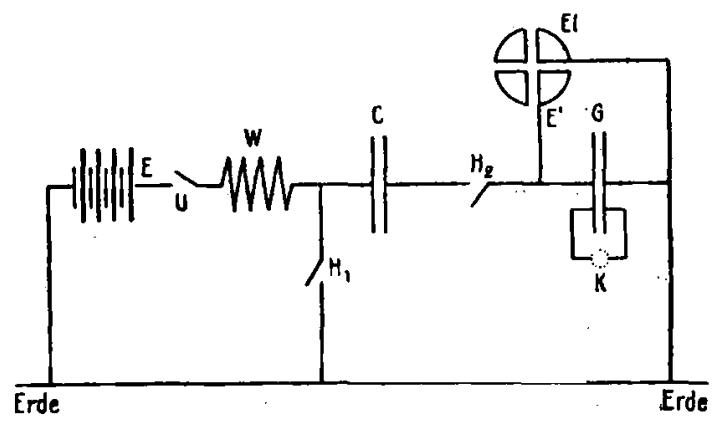

Fig. 1.

Bezeichnungen:

$C$ Versuchskondensator,

a Hilfskondensator.

E EMK. (Batterie),

$E^{\prime}$ das zu messende Potential an $G$,

$W$ Widerstand,

El Quadrantelektrometer,

$\left.\begin{array}{c}H_{1} \\ H_{2}\end{array}\right\}$ Kontakthebel,

K KurzsohluBstöpsel。

$U$ Unterbrecher.

Sie besteht:

a) aus der ,normalen" Ladung;

b) aus der gebildeten Rückstandsladung;

c) aus der Elektrizitätsmenge, die infolge Leitung durch $C$ hindurchgegangen ist.

Extrapolation auf ganz kurze Ladezeit liefert die „normale“, von Rückstand und Leitungseinflüssen freie Ladung, also auch die Kapazität $C_{\mathfrak{c}}$; Differentiation nach der Zeit die Stromstärke in jedem Znitmoment. Ist der Anteil strtionärer Leitfähigkeit am Strome bekannt, so ist es auf diese Weise möglich, den Nachladungsstrom als Funktion der Zeit zu finden.

Das Schaltungsschema möge kurz erläutert werden: Der Versuchskondensator $C$ und der viel größere Hilfskondensator $G$ sind hintereinander geschaltet und stehen durch den 
Widerstand $W$ rnit dem einen Pol einer Hochspannungsbatterie (Akkumalatoren, 100 Volt) in Verbindung. Der andere Pol der Butterie liegt an Erde. $H_{1}$, der erste Kontakthebel des Helmholtzpendels, befindet sich in der Leitung, die die eine Belegung des Versuchskondensators erdet; der zweite, $\mathrm{H}_{2}$, liegt in der Leitung zwischen Versuchskondensator und Hilfskondensator. Die freie Belegung des Hilfskondensators ist dauernd geerdet; der Hilfskondensator selbst kann durch einen Stöpsel $K$ kurzgeschlossen werden.

Bei der Ausführung der Versuche stellt man die Schlagstifte am Pendelquadranten bzw. den Schlitten mit der Mikrometerschraube auf ein bestimmtes Zeitintervall ein. Die Unterbrechungsstellen $H_{1}$ und $H_{2}$ sind geschlossen, und beide Kondensatoren besitzen das Potential der Erde. Dann wird die Batterie $E$ eingeschaltet, der Kurzschlußstöpsel $K$ weggenommen, und man überzeugt sich, daß das Elektrometer keinen Ausschlag gibt, somit keinerlei Rückstandsladungen in den Kondensatoren vorhanden sind; eine kleine Nullpunktsverschiebung wird notiert. Durch Ziehen an der Schnur löst man das Pendel aus. Beim Wegschlagen von $H_{1}$ beginnt die Aufladung des Kondensatorsystemes; beim Wegschlagen von $\mathrm{H}_{2}$ wird sie unterbrochen und der (ballistische) Ausschlag des Elektrometers miBt das Potential $E$ ', das auf $G$ erreicht worden ist. Dieses entspricht einer Elektrizitätsmenge

$$
e^{\prime}=E^{\prime} \cdot G,
$$

wenn mit $G$ zugleich auch die Kapazität des Hilfskondensators bezeichnet wird. Damit ist ein Punkt der Kurve, die die Ladung in Abhängigkeit von der Ladezeit gibt, aufgenommen. Die Batterie $E$ wird abgeschaltet, sämtliche Unterbrechungsstellen geschlossen und so die ganze Anordnung wieder geérdet. Nach 4-5 Minuten kann eine weitere Messung mit einem anderen Zeitintervall folgen. Zur Kontrolle and zur Erhöhung der Genauigkeit wurde jeder Punkt 2-3mal bestimmt.

\section{c) Korrektionen und Berechnung.}

Die Größenordnung der Kapazitäten $C$ und $G$ wird vor allem durch zwei Bedingungen festgelegt: es soll erstens die „,normale“ Ladung in kürzester Zeit vor sich gehen und ferner 
das Potential $E^{\prime}$ klein sein gegen das angelegte Potential $E$. Der Versuchskondensator steht dann, abgesehen von den jetzt zu besprechenden Korrektionen, eine genau gemessene Zeit unter bekannter konstanter Spannung; es besitzt die eine Kondensatorbelegung auch, wie es bei allen Wechselstrommessungen der Fall sein wird, nahezu das Potential der Firde.

Setzt $\operatorname{man} E=100 \mathrm{~V}, C=0,001 \mathrm{MF}, G=0,3 \mathrm{MF}$, so ist $E^{\prime}$ nach beendeter „normaler“ Laadung $0,33 \mathrm{~V}$ und wächst im Laufe des Versuches infolge Nachladung und Leitung noch um 20-30 Proz. Man rechnet daher genügend genau, wenn man für die Spannung am Versuchskondensator einsetzt:

$$
E_{\mathrm{C}}=\boldsymbol{E}^{\prime}-E^{\prime} \text {, }
$$

wo $E^{\prime}$ einen mittleren Wert von $E^{\prime}$ bedeuten soll. Die Größe dieser Korrektion beträgt $1 / 3$ Proz. Sie gilt natürlich nur nach erfolgter ,normaler" Ladung. Diese dauert a ber praktisch nicht länger als $7,0 \cdot 10^{-5}$ Sek., wie sich aus $C=0,001 \mathrm{MF}, G=0,3 \mathrm{MF}^{\prime}$ und $W=10000 \Omega$ berechnet. Ich sah davon $a b$, den Widerstand $W$ kleiner zu nehmen, da zur sicheren Konstanterhaltung der elektromotorischen Kraft $E$ der verwendeten KlingelfuBhochspannungs batterie ein geringer Stromverbrauch angestrelbt, wurde. Es ist aber zu berücksichtigen, daß infolge der endlichen Zeit, die die Spannung braucht, um am Kondensatorsystem ihren vollen Betrag zu erreichen, die gemessene Elektrizitätsmenge $e^{\prime}=E^{\prime} G$ etwas kleiner ist, als wenn die ganze Spannung $E$ schon rom Zeitmoment $t=0$ an wirksam gewesen wäre. Der Fehlbetrag ergibt sich aus der Überlegung. daß Nachladungs- und Leitungsstrom der Spannung am Versuchskondensator proportional sind, diese aber wie

$$
b\left(1-e^{-\frac{1}{W C} t}\right)
$$

wächst. Der Strom hat also die Form:

$$
i=E\left(1-e^{-\frac{1}{W C} t}\right) f(t)
$$

and für die Korrektion $\Delta e$ gilt bei finem Zeitintervall $t_{1}$

$$
\begin{gathered}
\int_{0}^{t_{1}} E\left(1-e^{-\frac{1}{w c} t}\right) f(t) d t+\Delta e=\int_{0}^{t_{1}} E f(t) d t \\
\Delta e=E \int_{0}^{t_{1}} e^{-\frac{1}{W c} t} \cdot f(t) d t \\
e=e^{\prime}+\Delta e .
\end{gathered}
$$


Solange $f(t)$ niclit bekannt ist, läBt sich $\Delta e$ nicht genau angeben; man weiß aber, daß für den Leitungsstrom $f(t)$ als Konstante anzusetzen ist und für den Nachladungsstrom. als eine Funktion, die bei großen Werten beginnt und mit der Leit abnimmt. Deshalb zerlegt man das Integral zweckmäßig in zwei Teile

$$
\Delta e=E \int_{u}^{t^{\prime}} e^{-\frac{1}{W^{2}} \cdot t} \cdot f(t) d t+E \int_{t^{\prime}}^{t_{1}} e^{-\frac{1}{W c} t} f(t) d t=\Delta e_{1}+\Delta e_{2}
$$

wo $t^{\prime}$ die sogenannte normale Ladungszeit des Kondensators sein soll, also praktisch etwa $7 \cdot W \cdot C$, und erkennt, daß für Zeiten $t_{1}>t^{\prime}$ der zweite Summand klein bleilt gegen den ersten. $\Delta e$ ist also nach abgelaufener normaler Ladung als merklich konstant zu betrachten. Dies ist insofern günstig, als bei der Berechnung des Stromes, den man durch die Billung der Differenzenquotienten aus $e$ erhält, die Korrektion $\Delta e$ gar nicht berücksichtigt $z u$ werden braucht, solange man T/eiten verwendet, die größer sind als $7,0 \cdot 10^{-5}$ Sek. Für den Fall eines Papierkondensators (vgl. p. 335 u. 347) von 0,001740 MF Kapazität berechnet sich z. B. unter der sicher ungünstigen Annahme $f(t)=B t^{-n}, \Delta e=4,32 \cdot 10^{-9}$ coul, während $e$ von der Größe $200 \cdot 10^{-9}$ coul ist, also $\Delta e / e=2$ Proz.

Es ist wichtig, daß der Hilfskondensator $G$ in hohem Maße idealen Anforderungen entspricht, insbesondere eine von der Ladezeit unathängige Kapazität besitzt. Es stand mir ein zwölfteiliger Präzisions-Glimmerkondensator von Siemens \& Halske zur Verfügung, mit einer Gesamtkapazität von 1,002 Mi' und einer kleinsten Kapazität von 0,00103 MF. Ich prüte ihn in der Weise, daB ich im Schaltungsschema I an Stelle von $C$ einen Luft-(Dreh-)Kondensator einschaltete und die Ladung von $G$ bei Änderung der Ladezeit von 0,001 bis 0,2 Sek. bestimmte. Beträgt bei einer Zunahme der Ladezeit um $\Delta t^{\prime}$ Sek. die Vergrößerung der Ladung $\Delta e^{\prime}$, so ist für die Korrektion maßgebend der Ausdruck $1 / e \cdot \Delta e^{\prime} \mid \Delta t^{\prime}$; ich fand für den Glimmerkondensator:

$$
\frac{1}{e} \cdot \frac{\Delta e^{\prime}}{\Delta t^{\prime}}=0,022 \mathrm{sec}^{-1} \text {. }
$$

Da die verwendeten Zeiten nie über 0,23 Sek. hinausgehen, bleibt diese Korrektion unter 0,5 Proz. 
Beiläufig sei bemerkt, daßB ich diese Anordnung zugleich benutzte, um die Kapazitüt les Drehkondensators für verschiedene Einstellungen desselben zu bestimmen; sie ist gegeben durch die Formol

$$
c=\frac{E^{\prime}}{E^{\prime}-E^{\prime}} G .
$$

Um bei der Messung des Potentials $E^{\prime}$ mit dem Elektrometer von Korrektionen möglichst unabhängig zu sein, wurde vor oder nach jeder Meßreihe das Elektrometer mit Hilfe eines Elementes von bekannter elektromotorischer Kraft und eines Spannungsteilers absolut geeicht, $d$. h. eine Kurve aufgenommen, die das Potential in Volts in Funktion der abgelesenen Ausschläge ergab. Das Elektrometer, ein Quadrantelektrometer nach Konstruktion von Prof. Dr. A. Klei$\mathrm{n} \theta \mathrm{r}$, ergab bei $40 \mathrm{~V}$ Nadelspannung und Ablesung mit Spiegel und Skala eine Empfindlichkeit von rund $800 \mathrm{~mm}$ pro Volt (Skalenabstand $2300 \mathrm{~mm}$ ). Fs erwies sich als notwendig, zur elektrostatischen Abschirmung und "zur Erzielung eindeutig definierter Kapazitäten, das Elektrometer, die Kondensatoren und alle Zuleitungen mit geerdeten Metallhüllen zu umgeben. Die Zuleitungen wurden moglichst kurz gewählt, so daB ihr Einfluß nicht in Betracht kam.

Ist nach Anbringung sämtlicher Korrektionen die Ladung $e$ in Abhängigkeit von der Ladezeit $t$ gefunden worden, so wird das der Beobachtung zu Giunde liegende Zeitintervall in Abschnitte ungefähr gleicher Zunahme von $e$ geteilt und es werden die Differenzenquotienten

$$
\frac{e_{2}-e_{1}}{t_{1}-t_{1}}=\frac{\Delta e}{\Delta t}
$$

gebildet. Sie ergeben den Gesamtstrom für die Zeit $\frac{t_{1}+t_{3}}{2}$, inshesondere für Zeiten nach erfolgter "normaler" Ladung die Summe von Nachladungs- und Leitungsstrom.

\section{Die Bestimmung der stationären Leitfähigkeit.}

Der stationäre Leitungsstrom kann mit einem empfindlichen Galvanometer gemessen werden. Ich zog jedoch eine Schaltung mit dem Helmholtzschen Pendel wegen ihrer größeren Empfindlichkeit vor, und. weil sie zugleich die Bequemlichkeit bot, durch geringe Abänderungen aus dem 
Schaltungsschema I hervorzugehen. Man ersparte sich so die Aufstellung und Eichung eines weiteren Instrumentes. Das Wesentliche ist aus der Zeichnung ersichtlich.

Sohaltungssohema II.

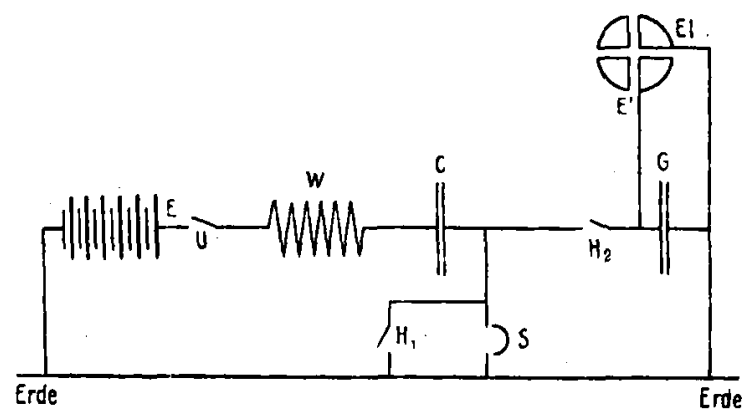

Fig. 2.

Bezeichnungen:

Größenordnung:

C Versuchskondensator,

G Hilfskondenkator,

$E$ EMK. (Batterie),

$E^{\prime}$ das zu messende Potential an $G$,

$W$ Widerstand,

0,001 bis $0,004 \mathrm{MF}$.

0,002 , 0,02 ,"

100 Volt

0,01 bis 0,5 Volt

$10^{4} \Omega$

El Quadrantelektrometer,

\begin{tabular}{c|c}
$H_{1}$ & Kontakthebel, \\
$H_{2}$ & \\
$U$ & Unterbrecher, \\
$S$ & ,"
\end{tabular}

Vor dem Versuche sind die beiden Kontakthebel $H_{1}$ und $H_{2}$ geschlossen, die Unterbrecher $U$ und $S$ geöffnet. Zur Zeit $t=0$ wird die Batterie eingeschaltet und es fließt ein Strom von der Batterie durch den Versuchskondensator $C$ zur Erde. Löst man nach gemessener Zeit die Fixierung des Pendels, so werden in einem Intervall von $\Delta t$ Sek. $H_{1}$ und $H_{2}$ kurz nacheinander weggeschlagen, während dessen die Elektrizitätsmenge

$$
e=G \cdot E^{\prime}
$$

auf den Hilfskondensator übergeht. Für die Stromstärke folgt daraus :

$$
i=\frac{e}{\Delta t} \text {. }
$$


Fiür $\Delta t=0,2$ Sek., $G=0,002 \mathrm{MF}, E^{\prime}=0,01 \mathrm{~V}$ erhält man $i=1,0 \cdot 10^{-10} \mathrm{~A}$, was noch mit einer Genauigkeit von 5 Proz. ausgemessen werden kann. Wenn nötig, kann man die Empfindlichkeit noch weiter treiben.

Auch hier hat man dafür zu sorgen, daß $E^{\prime}$ stets ein kleiner Bruchteil von $E$ bleibt, damit der Kondensator $C$ als unter konstanter Spannung stehend angesehen werden kann. Unmittelbar nach dem Offnen von $H_{2}$ muB der Unterbrecher $S$ geschlossen werden, damit kein dauernder Unterbruch des Stromes stattfindet. So ist es möglich, immerhin mit Berücksichtigung der kleinen Störungen, die das kurze Aufhören des Stromes verursacht, dessen Größe und Verlauf beliebige Zeiten nach Anlegen der Spannung $E$ zu ermitteln.

\section{Die Bestimmung des Wechselstromeffektverlustes.}

a) Die Sohaltung.

Zur Bestimmung der Wechselstromeffektverluste wählte ich eine direkte dynamometrische Messung, und zwar mit Hilfe von W. E. Sumpners ${ }^{1}$ ) Elektrodynamometer.

Es besteht aus einem hufeisenförmigen Elektromagneten $M$ von fein unterteiltem Weicheisen. Durch den Erregerstrom wird zwischen seinen Polen ein kräftiges, periodisch wechselndes Magnetfeld ergeugt, das durch einen Weicheisenkern verstärkt wird. In dem engen Luftraum zwischen Magnet und Eisenkern hängt die Drehspule $S$, durch die der zu messende Strom geschickt wird. Das Aufhängeband trägt einen kleinen Spiegel; die Ablesung geschieht mit Fernrohr und Skala. Erregerstrom und Meßstrom müssen absolut gleiche Periodenzahl haben, werden daher am besten derselben Wechselstromquelle entnommen.

Zur Messung kleiner Leistungen gibt W. E. Sumpner eine Schaltung an, bei der in den Meßstrom ein Transformator eingeschaltet wird. Dieses Hilfsmittel, das für genaue quantitative Messungen unzulässig ist, kann durch folgende Anordnung umgangen werden:

1) W. E. Sumpner u. W. Cs. Philipps, Proc. of Phys. Soo. of London 22; Phil. Mag. (6) 20. p. 309. 1910. 
Schaltungsschema III..

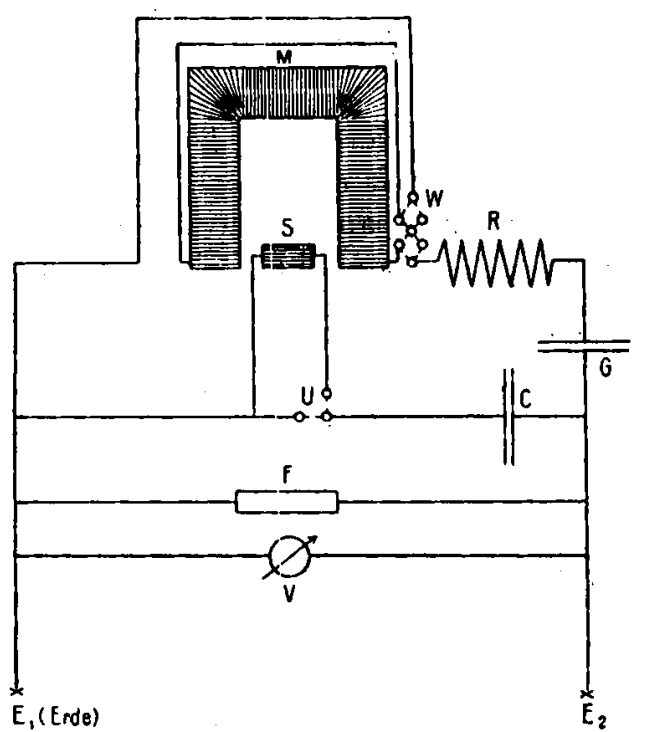

Bezeichnungen:

C Vcrsuchskondensator,

$G$ Hilfskondensator, $E_{1}-E_{2}$ Wechselstromquelle ( $E$ an Erde),

$\boldsymbol{R}$ Widerstand,

$M$ Elektromagnet,

$S$. Drehspule,

$W$ Wippe,

$U$ Umschalter,

$V$ Voltmeter,

$F$ Frequenzmesser, $E_{1} S C E_{2}$ MeBkreis, $E_{1} W M R G E_{8}$ Erregerkreis.

Größenordnung:

C: 0,001 bis 0,004 MF.

$G: \quad 0,9 \mathrm{MF}$.

E: 100 Volt, 50 Period.,

Fig. 3.

$R: 7000$ bis $10000 \Omega$

$E_{1}-E_{2}$ ist die Wechselstromquelle; sie dient sowohl für den Meßkreis, der die Versuchskapazität $C$, die Drehspule. $S$, sowie einen Umschalter $U$ enthält, als den Erregerkreis. Der Pol $E_{1}$ ist geerdet. Die Stromstärke im Erregerkreis wird durch Finstellen der Kapazität $G$ mit der Spannung in Resonanz gebracht und ihre Intensität durch den Widerstand $R$ reguliert. Da die Selbstinduktion des Elektromagneten sehr groß ist, ca. 11 Henry, so genügt eine Kapazität $G$ von ungefähr $0,9 \mathrm{MF}$ zur Herstellung der Resonanz. Ich verwendete den zwölfteiligen Glimmerkondensator von Sie mens \& Halske, der noch Tariationen von 1 Prom. gestattete.

Auf die Drehspule wirkt allgemein ein Drehmoment, das proportional ist au $H J \cos \alpha$, wenn mit $H$ und $J$ die Effektivwerte der magnetisıhen Feldstärke bzw. dẹs Meßstromes bezeichnet werden, mit $a$ ihre Phasendifferenz. Da $H$ mit dem Erregerstrom und sornit mit der Spannung in Phase ist, der Meßstrom jedoch eine Verschiebung von $\alpha=\pi / 2-\delta$ gegen diese hat, so folgt für das Drehmoment:

$$
D=\text { konst } \cdot H J \sin \delta \text {, }
$$


Daraus ersieht man, daB bei konstant bleibender Fffektivspannung das Drehmoment dem Fffektverlust in Meßkreis proportional ist, dern für diesen gilt:

$$
Q=E_{\text {erf. }} J_{\text {eff. }} \sin \delta \text {. }
$$

Das Elektrodynamometer funktioniert so als hochempfindliches Wattmeter und gibt Ausschläge, die der verbrauchten Leistung direkt proportional sind.

b) Die Empfindlichkeit.

Das Flektrodynamometer wurde absolut geeicht durch Herstellung eines bekannten Verlustes im Meßkreis vermittelst einer verlustlosen Kapazität (Drehkondensator) mit vorgeschaltetem, kapazitätsfreiem und selbstinduktionsfreiem Widerstand. Die Berechnung geschieht nach der Formel:

$$
Q=\omega^{2} E^{2} C^{2} W
$$

die hinreichend genau ist, da es sich ja nur um kleine Verlustwinkel handelt. $\omega$ ist die Kreisfrequenz, das $2 \pi$-fach $\theta$ der Periodenzahl, $E$ die Effektivspannung, $C$ die Kapazität und $W$ der Widerstanḍ. Da außer dem Widerstand alle Größen im Quadrat vorkommen, erfordern alle Instrumente eine sorgfältige Eichung. Als kapazitäts- und induktionsfreier Widerstand diente ein Widerstand von Siemens \& Halske; er besitzt fünf Abteilungen zu je ungefähr $200000 \Omega$, die nach Bedarf hintereinander oder parallel geschaltet werden können. Man erhält so einen Bereich von 50000-1000000 $\Omega$. Die Ergebnisse einer solchen Eichung sird z. B.:

\begin{tabular}{c|c|c}
\hline$W^{\prime} \Omega$ & $C^{M F}$ & Ergisec \\
\hline 248000 & 0,000256 & 8,54 \\
& 0,000529 & 8,55 \\
& 0,000808 & 8,49 \\
474950 & 0,000256 & 8,57 \\
& 0,000529 & 8,50 \\
719050 & 0,000256 & 8,52
\end{tabular}

$\varepsilon$ bedeutet die Empfindlichkeit in Erg/sec pro Skalenteil der Ablesung mit Spiegel und Skala. Der Drehkondensator ist nach dem'p. $\mathbf{3 1 5}$ angedeuteten Verfahren geeicht. Durch Kommutieren des Ausschlages kann diese Empfindlichkeit noch verdoppelt werden. 
Es ist so möglich, auch bei verhältnismäBig niedrigen Spannungen und Frequenzen noch genaue Bestimmungen der dielektrischen Verluste zu machen. Man entgeht zugleich den Fehlerquellen, die die Messungen mit hohen Spannungen und Frequenzen mit sich bringen. Es möge die Leisturggfähigkeit dieser Methode mit derjenigen anderer Methoden an einem Beispiel kurz zahlenmäBig verglichen werden.

Ein Plattenkondensator (vgl. p. 339), bestehend aus zwei kreisförmigen Platten von 12,50 cm Radius mit dem Dielektrikum Glas (Dicke 0,215 cm), ergab bei einer Beanspruchung mit Wechselstrom von rund 100 Volt und 50 Perioden einen Effektverlust von rund $1000 \mathrm{Erg} / \mathrm{sec}$, ausgemessen mit einem mittleren Fehler von' 5 Proz. Nimmt man den Verlust als der Frequenz und dem Quadrat der Spannung proportional an ${ }^{1}$ ), so würde er bei 10000 Volt und 50 Perioden 1,0 Watt betragen. Diese Finergiemenge würde in 5 Minuten, Wärmeverluste durch Ableitung nicht gerechnet, das Dielektrikum um ca. $0,14^{\circ} \mathrm{C}$ erwärmen, könnte also auch kaum genauer als auf 5 Proz. bisstimmt werden. Um bei 100 Volt in der gleichen Zeit dieselbe Temperaturerhöhung zu erhalten, müßte die Frequenz auf 500000 Perioden in der Sekunde gesteigert werden.

Die neueren Messungen über dielektrische Verluste sind meistens, solange es sich nicht um sehr hohe Frequenzen handelt, mit der Wechselstrombrücke gemacht worden. Dieses Verfahren läßt eine sehr genaue Bestimmung der Kapazität und des sogenannten Verlustwinkels $\delta$, der Phasenabweichung des Stromes von der durch einen idealen Kondensator bedingten Phase, zu. ${ }^{2}$ ) Ich wählte es für meinen Zweck nicht, um von der Berechnung des Effektverlustes für bestimmte Spannung aus Kapazität und Verlustwinkel frei zu sein; ferner dürften die direktien Messungen mit dem Elektrodynnmometer rascher und bequemer vor sich gehen. Der erwähnte Kondensator besaf eine Kapazität von 0,001530 MF. Aus dem bekannten Effektverlust von $1000 \mathrm{Erg} / \mathrm{sec}$, der Spannung von 100 Volt und der Periodenzahl 50 berechnet sich

1) Vgl. z. B. B. Monasch, Ann. d. Phys. 22. p. 905. 1907; ferner H. Jordan, 1. c.

2) Vgl. z. B. K. W. Wagner u. A. Wertheimer, Physik. Zeitschr. 18. p. 368. 1912 . 
der Verlustwinkel zu $1^{\circ} 10^{\prime}$. Diese Genauigkeit dürfte der in der Brücke erreichbaren nahe kommen.

Die Empfindlichkeit läßt sich durch den im Erregerkreise angebrachten Widerstand $R$ in weiten Grenzen rerändern. Ich benutzte sie meistens in einer Größe von 8-12 Erg/sec pro Skalenteil; doch läBt sie sich bei Verwendung sehr konstanten Wechselstromes wohl noch weiter steigern.

\section{o) Fehlerquellen und Verbesserungen.}

Die Effektmessung mit dem Sumpnerschen Elektrodynamometer in Resonanzschaltung wird nur dann zuverlässig, wenn Wechselstrom konstanter Frequenz und Spannung verwendet werden kann. $\mathrm{Da} \mathrm{mir}$ einzig das städtische Lichtnetz zur Verfügung stand, so war die MeBgenauigkeit durch die Spannungs- und Frequenzschwankungen desselben beeinträchtigt. Mit einem Voltmeter $(V)$ und einem Frequenzmesser $(F)$ wurde der Gang dieser Schwankungen kontrolliert. Wählte man eine günstige Tageszeit, so pflegten sie 1 Proz. kaum zu übersteigen. - Zur Frhöhung der Genauigkeit wurde ein ballistisches Verfahren angewendet. Durch einen Umschalter $U$ (vgl. Schaltungsschema III) wurde das Elektrodynamometer kurz geschlossen and der Meßstrom direkt zur Erde geleitet, zur Messung der Umschalter gekehrt, was vom Platz des Beobachters aus geschehen konnte, so daß nun der Strom die Drehspule $S$ passierte. Der entstehende ballistische Ausschlag ergab ein genau proportionales MaB für den Effektverlust. Da die Schwankungen mehr zufälliger Natur sind und in kürzeren Zeiten um denselben Betrag sich herumbewegen, auf den die Resonanzeinstellung gemacht wird, so ergibt das Mittel aus einigen Beobachtungen einen genügend zuverlässigen Wert. Ferner kann der Erregerstrom durch die Wippe $W$ kommutiert werden; dadurch erhält man Ausschläge nach rechts und nach links, was die Nullpunktsfehler eliminiert. Von der Anbringung einer Wippe im Kreise des Meßstromes sah ich $a b$, um die Leitungen zu verkürzen und möglichst kapazitätsfrei und induktionsfrei zu behalten.

Den größten Fehler bedingt eine falsche Resonanzeinstellung, da er sich durch Kommutieren der Ströme nicht eliminieren läBt. Die Effektmessung wurde daher so vorgenommen, daß nach je einer Gruppe von Ablesungen am Elektrodynamo- 
meter die Resonanzstellung nachkontrolliert wurde. Der Versuchskondensator wurde mit einem verlustlosen Drehkondensator vertauscht und die Hiltskapazitït $G$ so reguliert, daß bei beliebiger Kapazität des Drehkondensators das Elektrodynamometer keinen Ausschlag gab. Damit sind zugleich die kleinen Phasenverschiebungen, dio die Hysteresis im Eisen des Elektromagneten, die geringe Selbstinduktion der Drehspule und deren Widerstand (50 $\Omega$ ) verursachen, korrigiert. Die Drehspule erfordert eine sorgfältige Einstellung; sie muß so im Magnetfeld hängen, da $B$ sie auch bei sehr starkem Felde sich nicht bewegt, wenn man die Zuführungsklemmen kurzschließt. Es ist darauf zu achten, daB die Instrumente so geschaltet werden, wie im Schaltungsschema III angegeben ist; ihre Kapazität gegen Erde wird dadurch möglichst ohne Einfluß gemacht. Insbesondere soll beim Elektrodynamometer eine Klemme des Erregerkreises und eine Zuführungsklemme der Drehspule an Erde liegen.

Die Methode der absoluten Eichung habe ich schon erwähnt. Im Verlaufe jeder Meßreihe wurde eine solche Eichung ausgeführt, dabei der Drehkondensator möglichst auf die Kupazität des .Versuchskondensators eingestellt; auf diese Weise fallen auch Fehler in der Resonanzeinstellung fast ganz heraus.

\section{Die Versuchsresultate.}

\section{Feste Dielektriken.}

a) Der Verlauf des Naohladungsstromes. Stationäre Leitfähigkeit.

Die Untersuchung erstreckte sich auf feste und flüssige Dielektriken. In der Auswahl fester Dielektriken war ich dadurch beschränkt, daß sie, um eine zuverlässige Messung zu ermöglichen, die Eigenschaft besitzen mußten, in kurzer Zeit schon beträchtliche Rückstände zu bilden. Diese Forderung erfüllten nur inprägnierte Papiere und Glas in ausreichendem Maße. ${ }^{1}$ ) Für diese Dielektriken ist eine Anzahl Messungen mit möglichster Sorgfalt durchgeführt worden.

Verhältnismäßig große Rückstandsbildung in kurzer Zeit und sehr langsames Anwachsen des Rückstandes bei späteren

1) Vgl. C. L. B. Schuddemagen, Proc. Amer. Acad. 44. p. 465. 1909. 
Zeiten ist nach der Wàgnerschen Theorie der unvollkommenen Dielektriken ein Kennzeichen kleiner Verteilungskonstante , (also weitgehender Zerstreuung der Zeitkonstanten). Ia in diesem Falle innerhalb eines beschränkten Bereiches der Nachladungsstrom dargestellt werden kann durch die Gleichung (7) bzw. $\left(7^{\prime}\right)$ :

$$
i=E C_{0} \beta t^{-n}=B t^{-n}
$$

wobei $n=1+2 b^{2} \log$ nat $\tau / T_{0}$ (vgl. p. 314) nicht stark von Eins abweicht, so lag es nahe, vor allem die Anwendbarkeit dieser Gleichung auf den experimentell ermittelten Nachladungsstrom $7 u$ versuchen. Man hat den Vorteil, daß man mit nur zwei Materialkonstanten $\beta$ und $n$ auskommt, und da $\beta$ bei cler Berechnung der Wechselstromverluste die vorkommenden Integrale (vgl. p. 317) sich leicht auswerten lassen. Wie weit diese Näherung brauchbar ist, ergibt dann ein Vergleich der berechneten Stromstärken bzw. Wechselstromverluste mit den gemessenen. Durch Logarithmieren de: obigen Gleichung erhält man

$$
\log i=\log B-n \log t,
$$

woraus durch Bildung der Differenzquotienten hervorgeht

$$
\frac{\Delta \log i}{\Delta \log t}=-n \text {. }
$$

Diese Beziehung, angewendet auf die einzelnen Intervalle des beobachteten Zeitraumes, ergibt die Probe der Formel. Aus bekanntem $n$ wird dann unmittelbar $B$ erhalten.

Die Versuchsresultate sind im folgenden in T'abellen mitgeteilt. Neben den unmittelbar aus den experimentellen Bestimmungen hergeleiteten Größen, der Ladung $e$ und der Stromstärke $i$ (vgl. p. 325), sind die nach der Formel berechneten Werte der Stromstärke $i_{\text {ber. }}$ angegeben, sowie die aus drei aufeinanderfolgenden Beobachtungen ermittelte Zahl $n$. Die erste Kolonne der Zeiten $t$ bezieht sich auf die Einstellung an der Mikrometerschraube bzw. am Pendelquadranten, gibt also die zar Ladung e gehörige Ladezeit; die zweite Kolonne der Zeiten $t^{\prime}$ gilt für die Stromstärken $i$. Das Verhältnis der gebildeten Rückstandsladung zur ,normalen“ Ladung geht aus der mit $\Delta C / C_{0}$ bezeichneten Kolonne hervor. $\triangle C$ bedeutet die 7unahme der Kapazität infolge der Rückstandsbildung, $C_{0}$ die auf unendlich kurze Ladezeit extrapolierte 
Kapazität. Insbesondere wurde noch die Kapazitätsvergrößerung $A C_{\infty}$ bei sehr großer Ladezeit (300 Sek.) ermittelt. Es geschah dies durch Vergleich der Versuchskapazitäten mit einem rückstandsfreien Luft-(Dreh-)Kondensator bekannter Kapazität am ballistischen Galvanometer.

Da der Nachladungsstrom erst durch Subtraktion des stationären Leitungsstromes von dem Strome, den man aus dem Zuwachs der Ladung erhält, hervorgeht, sind bei jedem Beispiel noch die Resultate der. Messungen nach Schaltungsschema II in einer kleinen 'Tabelle beigegeben.

\section{Bezeichnungen:}
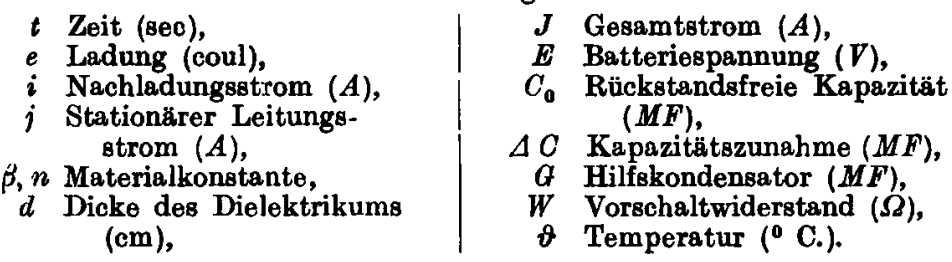

Kondeneator A.

Eine Lage paraffiniertas Seidenpapier; Herstellung des hiesigen Instituts.

a) Tabelle für den Nachladungsstrom. (Schaltungsschema I.)

\begin{tabular}{|c|c|c|c|c|c|c|}
\hline $\begin{array}{r}E=104, \\
\xi=16\end{array}$ & & $\begin{aligned} G=0,300 \mathrm{MF} \\
\beta=0,0511\end{aligned}$ & & & $C_{0}=$ & $58 \mathrm{Mr}$ \\
\hline$t \mathrm{sec}$ & $t^{\prime} \sec$ & $e$ coul & $i$ & $n$ & $i_{\text {ter. }}$ & $\frac{\Delta C}{C_{0}}$ \\
\hline
\end{tabular}

Zeitmessung mit der Mikrometerschraube:

\begin{tabular}{l|l|l|l|l||l|l|}
$0,328 \cdot 10^{-9}$ & $0,820 \cdot 10^{-3}$ & $1,206 \cdot 10^{-7}$ & $16,60 \cdot 10^{-7}$ & 0,80 & $16,63 \cdot 10^{-7}$ & 0,004 \\
1,312 & 1,804 & 1,222 & 8,79 & 0,017 \\
2,296 & 2,788 & 1,231 & 6,40 & $\mathbf{0 , 7 3}$ & $\mathbf{6 , 3 9}$ & 0,025 \\
$\mathbf{3 , 2 8 0}$ & $\mathbf{3 , 7 7 2}$ & $\mathbf{1 , 2 3 7}$ & $\mathbf{5 , 0 0}$ & $\mathbf{0 , 8 3}$ & $\mathbf{4 , 9 8}$ & $\mathbf{0 , 0 3 0}$ \\
$\mathbf{4 , 2 6 4}$ & & 1,242 & &
\end{tabular}

Zeitmessung mit dem Pendelquadranten:

\begin{tabular}{|c|c|c|c|c|c|c|}
\hline $\begin{array}{l}0,00233 \\
0,00467 \\
0,00937 \\
0,01407 \\
0,02347 \\
0,04695 \\
0,07045 \\
0,0946 \\
0,1440 \\
0,2265\end{array}$ & $\begin{array}{l}0,00350 \\
0,00702 \\
0,01172 \\
0,01877 \\
0,03521 \\
0,05870 \\
0,0825 \\
0,1193 \\
0,1852\end{array}$ & $\mid \begin{array}{l}1,232 \cdot 10^{-7} \\
1,244 \\
1,259 \\
1,268 \\
1,282 \\
1,302 \\
1,315 \\
1,326 \\
1,342 \\
1,361\end{array}$ & $\begin{array}{l}5,19 \cdot 10^{-7} \\
3,06 \\
2,05 \\
1,409 \\
0,856 \\
0,574 \\
0,439 \\
0,328 \\
0,229\end{array}$ & $\begin{array}{l}0,76 \\
0,78 \\
0,80 \\
0,79 \\
0,78 \\
0,77 \\
0,79 \\
0,82\end{array}$ & $\begin{array}{l}5,24 \cdot 10^{-7} \\
3,04 \\
2,04 \\
1,403 \\
0,859 \\
0,573 \\
0,438 \\
0,327 \\
0,231\end{array}$ & $\begin{array}{l}0,026 \\
0,036 \\
0,048 \\
0,056 \\
0,068 \\
0,084 \\
0,095 \\
0,104 \\
0,117 \\
0,133\end{array}$ \\
\hline 300 & & & & & & 0,75 \\
\hline
\end{tabular}


Dielektrisch̉e Effektverluste von Kondensatoren usw. $\quad 335$

b) Tabelle für den Leitungsstrom.

(Schsltungsschema II.)

$$
\begin{aligned}
& E=104,1 \mathrm{~V} \quad Q t=0,0100 \mathrm{MF} \quad \Delta t=0,1440 \mathrm{sec} \\
& \vartheta=16,5^{\circ} \mathrm{C} \text {. }
\end{aligned}
$$

\begin{tabular}{r|r|r}
\hline \hline$t$ sec & $e$ coul & $j$ \\
\hline \hline 15 & $1430 \cdot 10^{-12}$ & $99 \cdot 10^{-10}$ \\
135 & $7,8 \cdot 10^{-12}$ & $0,54 \cdot 10^{-10}$ \\
120 & $2,6 \cdot 10^{-12}$ & $0,18 \cdot 10^{-10}$
\end{tabular}

Ein Strom, entsprechend stationärer Leitfähigkeit, wird nicht erreicht. Auf jeden Fall verschwindet er während der ersten Sekunden vollständig gegen den Nachladungsstrom.

\section{Kondensator $\mathbf{B}$.}

Imprägniertes technisches Isolierpapier; Kreisplatten Kondensator $(r=12,5 \mathrm{~cm})$ mit zwei Lagen Papier; $d=0,040 \mathrm{~cm}$.

a) Tabelle für den Nachladungsstrom (Schaltungsschema I).

\begin{tabular}{|c|c|c|c|c|c|c|}
\hline$t \mathrm{sec}$ & $t^{\prime}$ sec & e coul & $i$ & $n$ & $i_{\text {ber. }}$ & $\frac{\Delta C}{C_{0}}$ \\
\hline
\end{tabular}

$$
\begin{array}{ccr}
E=103,9 \mathrm{~V} & a=0,502 \mathrm{MF} & W=10000 \Omega \\
& C_{0}=0,001740 \mathrm{MF} & \\
\vartheta=16,0^{\circ} \mathrm{C} . & \beta=0,158 & n=0,747
\end{array}
$$

\begin{tabular}{|c|c|c|c|c|c|c|}
\hline $\begin{array}{l}0,328 \cdot 10^{-} \\
1,312 \\
2,296 \\
3,280 \\
4,264\end{array}$ & $\begin{array}{l}0,820 \cdot 10^{-9} \\
1,804 \\
2,788 \\
3,772\end{array}$ & $\mid \begin{array}{l}1,820 \cdot 10^{-} \\
1,884 \\
1,920 \\
1,945 \\
1,966\end{array}$ & $\begin{array}{l}65,6 \cdot 10^{-7} \\
35,8 \\
26,0 \\
20,9\end{array}$ & $\begin{array}{l}\mathbf{0 , 7 7} \\
0,73 \\
\mathbf{0 , 7 3}\end{array}$ & $\begin{array}{l}65,3 \cdot 10^{-7} \\
36,0 \\
26,1 \\
20,8\end{array}$ & $\begin{array}{l}0,0 \\
0,0 \\
0,0 \\
0,\end{array}$ \\
\hline
\end{tabular}

\begin{tabular}{|c|c|c|c|c|c|c|}
\hline 0,00233 & \multirow{10}{*}{$\begin{array}{l}0,00350 \\
0,00702 \\
0,01172 \\
0,01877 \\
0,03521 \\
0,0587 \\
0,0825 \\
0,01193 \\
0,1852\end{array}$} & \multirow{10}{*}{$\mid \begin{array}{l}\mathbf{1 , 9 2 3} \cdot 10^{-2} \\
\mathbf{1 , 9 7 5} \\
\mathbf{2 , 0 3 6} \\
\mathbf{2 , 0 7 8} \\
\mathbf{2 , 1 3 7} \\
\mathbf{2 , 2 2 8} \\
\mathbf{2 , 2 9 2} \\
\mathbf{2 , 3 4 2} \\
\mathbf{2 , 4 2 0} \\
\mathbf{2 , 5 1 4}\end{array}$} & \multirow{10}{*}{$\begin{array}{c}21,9 \cdot 10^{-7} \\
13,1 \\
\mathbf{8 , 8 4} \\
\mathbf{6 , 2 4} \\
\mathbf{3 , 8 9} \\
2,70 \\
2,07 \\
1,58 \\
1,14\end{array}$} & \multirow{10}{*}{$\begin{array}{l}\mathbf{0 , 7 5} \\
0,76 \\
0,74 \\
0,75 \\
0,72 \\
0,78 \\
0,74 \\
0,75\end{array}$} & \multirow{10}{*}{$\begin{array}{c}21,8 \cdot 10^{-7} \\
13,0 \\
8,90 \\
6,26 \\
3,92 \\
2,68 \\
2,08 \\
1,57 \\
1,13\end{array}$} & 0,068 \\
\hline 0,00467 & & & & & & 0,097 \\
\hline 0,00937 & & & & & & 0,131 \\
\hline 0,01407 & & & & & & 0,154 \\
\hline 0,02347 & & & & & & 0,18 \\
\hline 0,0470 & & & & & & 0,238 \\
\hline 0,0704 & & & & & & 0,273 \\
\hline $\begin{array}{l}0,0946 \\
0,1440\end{array}$ & & & & & & \\
\hline & & & & & & \\
\hline 300 & & & & & & 1,38 \\
\hline
\end{tabular}

Zeitmessung mit der Mikrometerschraube:

Zeitmessung mit dem Pendelquadranten: 
b) Tabelle für den Leitungsstrom.

(Sohaltungsschema II.)

$$
\begin{gathered}
E=103,9 \mathrm{~V} \quad \begin{array}{c}
\quad t=0,0200 \mathrm{MF} \\
\vartheta
\end{array} \quad \Delta t=0,16,5^{\circ} \mathrm{C} .
\end{gathered}
$$

\begin{tabular}{c|c|c}
\hline$t$ sec & coul & $j$ \\
\hline 60 & $121,6 \cdot 10^{-12}$ & $9,1 \cdot 10^{-10}$ \\
180 & $70,2 \cdot 10^{-12}$ & $4,9 \cdot 10^{-10}$ \\
300 & $55,6 \cdot 10^{-12}$ & $3,9 \cdot 10^{-10}$ \\
900 & $2,9 \cdot 10^{-12}$ & $0,2 \cdot 10^{-10}$
\end{tabular}

Eine strtionäre Leitfähigkeit wird auch hier nicht erreicht; der Strom scheint mit der /heit fortwährend ab. zunehmen.

\section{Kondensator $\mathbf{C}$.}

Imprägniertes Seidenpapier, Teil aus einer Fernsprechkapazität von $2 \mathbf{M F}$, als Band von ca. $20 \mathrm{~cm}$ Länge und $4 \mathrm{~cm}$ Breite auf einen Paraffinkern gewiokelt und in Paraffin eingegossen.

a) Tabelle für den Nachladungsstrom.

(Schaltungsschema I.)

$$
\begin{array}{crc}
E=104,1 \mathrm{~V} & \theta=1,002 \mathrm{MF} & W=10000 \Omega \\
& C_{0}=0,00366 \mathrm{MF} & \\
\vartheta=17,0^{\circ} \mathrm{C} . & \beta=0,226 & n=0,785
\end{array}
$$

\begin{tabular}{|c|c|c|c|c|c|c|c|}
\hline & & & & & & & \\
\hline 0,00233 & 0,00350 & $3,960 \cdot 10^{-7}$ & $\left|75,5 \cdot 10^{-7}\right|$ & $75,0 \cdot 10^{-7}$ & & $70,4 \cdot 10^{-7}$ & 0,045 \\
\hline 0,00467 & 0,00702 & 4,139 & 48,0 & 47,5 & 0,66 & 40,8 & 0,092 \\
\hline 0,00937 & 0,01172 & 4,352 & 28,7 & 28,2 & 0,90 & 27,4 & 0,146 \\
\hline 0,01407 & 0,01877 & 4,477 & 18,09 & 17,62 & $\begin{array}{l}0,80 \\
0,60\end{array}$ & 18,9 & 0,181 \\
\hline 0,02347 & 0,03521 & 4 & 12,04 & 11.57 & 0,95 & 11,5 & 0,226 \\
\hline $\begin{array}{l}0,0470 \\
0,0704\end{array}$ & 0,0587 & 4,922 & 7,43 & 6,96 & 0,73 & 7,76 & 0,295 \\
\hline 0,0704 & 0,0825 & 5,087 & 5,90 & 5,42 & 0,64 & 5,92 & 0,042 \\
\hline, 0946 & 0,01193 & 5,242 & 4,75 & 4,28 & 0.56 & 4,43 & 0,378 \\
\hline 0,1440 & 0,1852 & $\begin{array}{l}0,502 \\
5,610\end{array}$ & 3,81 & 3,34 & & 3,13 & 0,585 \\
\hline 30 & & & & & & & $\mathbf{1 , 5 3}$ \\
\hline
\end{tabular}

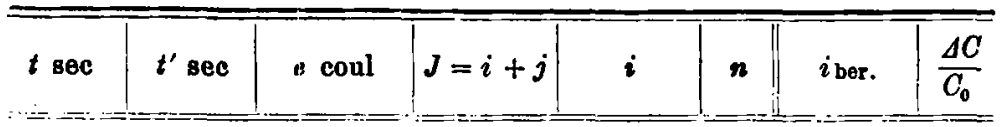

Zeitmessung mit dem Pendelquadranten: 
b) Tabelle fitr den Loitungsstrom.

(Schaltungssohema II.)

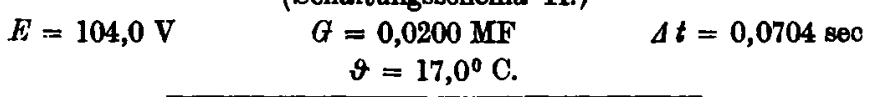

\begin{tabular}{r|l|l}
\hline$t$ sec & \multicolumn{1}{|c|}{$e$ coul } & $j$ \\
\hline 600 & $33,0 \cdot 10^{-10}$ & $4,7 \cdot 10^{-8}$ \\
900 & 32,8 & 4,7 \\
1200 & 32,7 & 4,7 \\
1500 & 33,0 & 4,7 \\
1800 & 33,0 & 4,7
\end{tabular}

In Gegensatz zu den beiden vorhergehenden Beispielen, bei denen kein stationärer Leitungsstrom aufzufinden war, und bei denen der Nachlidungsstrom sich wirklich gut nach der Gleichung $i=E C_{0} \beta t^{-n}$ darstellen läBt, zeigt dieser Kondensator Unregelmäßigkeiten. Die Werte von $n$ scheinen mit der Zeit abzunehmen, und zwar beträchtlich; es tritt ferner ein stationärer Leitungsstrom auf, der schon nach 0,1 Sek. ungefähr 10 Proz. des Nachladungsstromes ausmacht. Da man von diesem nicht weib, ob er wirklich von Anfang an stationär ist, oder vielleicht mit höheren Werten beginnt, wird die ganze Berechnung einigermaßen unsicher. Er entspricht jedoch einer Leitfähigkeit, die in die an Dielektriken beobachtete Größenordnung noch hineinpaBt. Setzt man die Dielektrizitätskonstante schätzungsweise gleich 2, so berechnet man aus der bekannten Kapazität und dem Widerstand

$$
\lambda=2,2 \cdot 10^{-14} \mathrm{~cm}^{-1} \Omega^{-1} \text {. }
$$

Kondensator D.

Leidener Flasche. $d=0,25 \mathrm{~cm}$.

a) Tabelle für den Nachladungsstrom.

(Schaltungsschema I.)

\begin{tabular}{|c|c|c|c|c|c|c|c|}
\hline$t \mathrm{sec}$ & $t^{\prime}$ sec & e coul & $J=i+j$ & $i$ & $n$ & $i_{\text {ber. }}$ & $\frac{\Delta C}{C_{0}}$ \\
\hline
\end{tabular}

$$
\begin{gathered}
E=103,8 \mathrm{~V} \quad G=1,002 \mathrm{MF} \quad W=10000 \Omega \quad C_{0}=0,00419 \mathrm{MF} \\
\vartheta=16,4^{\circ} \mathrm{C} . \quad n=0,662 \quad \beta=0,1352
\end{gathered}
$$

\begin{tabular}{|c|c|c|c|c|c|c|c|}
\hline $\begin{array}{l}0,328 \cdot 10^{-9} \\
0,1312 \\
2,296 \\
3,280 \\
4,264\end{array}$ & $\begin{array}{l}0,820 \cdot 10^{-} \\
1,804 \\
\mathbf{2 , 7 8 8} \\
\mathbf{3 , 7 7 2}\end{array}$ & $\left\{\begin{array}{l}4,357 \cdot 10^{-7} \\
4,421 \\
4,459 \\
4,487 \\
4,511\end{array}\right.$ & $\mid \begin{array}{l}64,9 \cdot 10^{-7} \\
38,5 \\
29,1 \\
23,8\end{array}$ & $\begin{array}{l}64,7 \cdot 10^{-7} \\
38,4 \\
28,9 \\
23,6\end{array}$ & $\begin{array}{l}0,66 \\
0,64 \\
0,67\end{array}$ & $\begin{array}{l}64,6 \cdot 10^{-7} \\
38,6 \\
28,7 \\
23,7\end{array}$ & $\begin{array}{l}0,0035 \\
0,018 \\
0,027 \\
0,033 \\
0,039\end{array}$ \\
\hline
\end{tabular}

Zeitmessung mit der Mikrometerschraube: 
(Fortsetzuug.)

\begin{tabular}{l|l|l|l|l|l|l|l|}
\hline $\sec$ & $t^{\prime} \sec$ & & & \\
\hline
\end{tabular}

Zeitmessung mit dem Pendelquadranten:

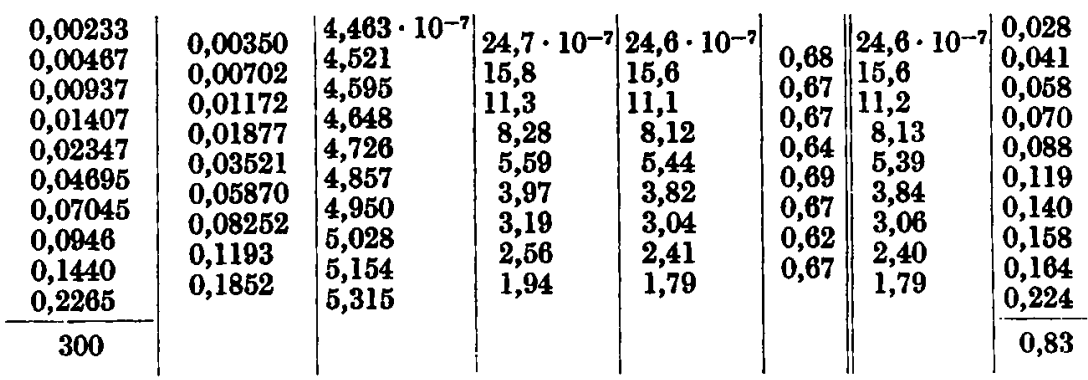

b) Tabelle für den Leitungsstrom.

(Schsitungssehema II.)

$$
\begin{aligned}
& E=103,8 \mathrm{~V} \quad G=0,0200 \mathrm{MF} \quad \Delta t=0,1440 \mathrm{sec} \\
& \vartheta=17,0^{\circ} \mathrm{C} \text {. }
\end{aligned}
$$

\begin{tabular}{c|l|l}
\hline$t$ sec & \multicolumn{1}{|c}{ coul } & \multicolumn{1}{|c}{$j$} \\
\hline 120 & $2,49 \cdot 10^{-\theta}$ & $0,173 \cdot 10^{-7}$ \\
210 & 2,28 & 0,158 \\
730 & 2,21 & 0,153 \\
1080 & 2,22 & 0,154 \\
1800 & 2,16 & 0,160
\end{tabular}

Da $j$ sich für die größeren Zeiten nicht mehr meBbar ändert, ist hier stationäre Leitung anzunehmen. Setzt man die Dielektrizitätskonstante des Glases gleich 6, so erhält man eine Leitfähigkeit $\lambda=1,83 \cdot 10^{-14} \mathrm{~cm}^{-1} \Omega^{-1}$, die z. B. mit den Angaben von J. $\mathrm{Curie}^{1}$ ) ungefähr übereinstimmt.

1) J. Curie, Aun. chim. phys. (6) 18. p. 203.1889. 
Dielektrische Effektverluste von Kondensatoren usw. $\quad 399$

\section{Kondensator $\mathbf{D}$.}

Glas $(d=0,215 \mathrm{~cm})$; Kreisplattenkondensator $(r=12,5 \mathrm{~cm})$.

a) Tabelle für den Nachladungastrom.

(Schaltungsschema $I_{\text {.) }}$

\begin{tabular}{|c|c|c|c|c|c|c|}
\hline & $\begin{array}{l}103,4 \mathrm{~V} \\
15,7^{\circ} \mathrm{C}\end{array}$ & & $\begin{array}{l}=0,300 \mathrm{M} \\
=0,00153 \\
=0,1148\end{array}$ & $W=$ & $100 \Omega$ & \\
\hline$t \mathrm{sec}$ & $t^{\prime} \sec$ & - coul & $J=i+j$ & $n$ & $i_{\text {ber. }}$ & $\frac{\Delta O}{C_{0}}$ \\
\hline
\end{tabular}

Zeitmessung mit der Mikrometerschraube:

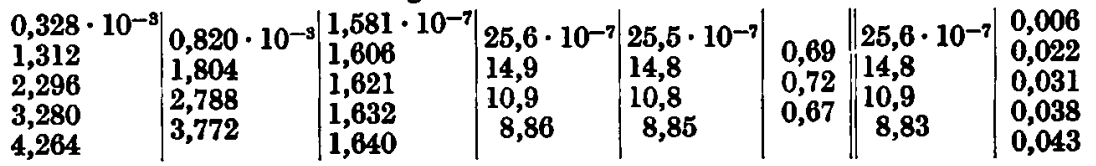

Zeitmessung mit dem Pendelquadranten:

\begin{tabular}{|c|c|c|c|c|c|c|c|}
\hline $\begin{array}{l}0,00233 \\
0,00467 \\
0,00937 \\
0,01407 \\
0,02347 \\
0,04695 \\
0,07045 \\
0,0946 \\
0,1440 \\
0,2265\end{array}$ & $\begin{array}{l}0,00350 \\
0,00702 \\
0,01172 \\
0,01877 \\
0,03521 \\
0,05870 \\
0,08252 \\
0,1193 \\
0,1852\end{array}$ & $\mid \begin{array}{l}1,622 \cdot 10^{-7} \\
1,644 \\
1,671 \\
1,690 \\
1,717 \\
1,761 \\
1,792 \\
1,817 \\
1,856 \\
1,905\end{array}$ & $\mid \begin{array}{l}9,29 \cdot 10^{-7} \\
5,76 \\
3,99 \\
2,87 \\
1,87 \\
1,31 \\
1,040 \\
0,803 \\
0,592\end{array}$ & $\begin{array}{l}9,28 \cdot 10^{-7} \\
5,75 \\
3,98 \\
2,86 \\
1,86 \\
1,30 \\
1,032 \\
0,795 \\
0,584\end{array}$ & $\begin{array}{l}0,69 \\
0,72 \\
0,70 \\
0,69 \\
0,70 \\
0,68 \\
0,70 \\
0,71\end{array}$ & $\begin{array}{l}9,24 \cdot 10^{-2} \\
5,71 \\
4,01 \\
2,88 \\
1,86 \\
1,31 \\
1,03 \\
0,796 \\
0,584\end{array}$ & $\begin{array}{l}0,032 \\
0,046 \\
0,063 \\
0,075 \\
0,092 \\
0,120 \\
0,140 \\
0,156 \\
0,181 \\
0,212\end{array}$ \\
\hline 300 & & & & & & & 0,78 \\
\hline
\end{tabular}

b) Tabelle für den Leitungsstrom.

(Schaltungsschema II.)

$$
\begin{array}{ll}
E=103,3 \mathrm{~V} & G=0,0100 \mathrm{MF} \\
\vartheta=15,9^{\circ} \mathrm{C} . & \Delta t=0,1440 \mathrm{sec}
\end{array}
$$

\begin{tabular}{r|l|l}
\hline$t$ sec & $e$ coul & $j$ \\
\hline 180 & $8,10 \cdot 10^{-10}$ & $22,1 \cdot 10^{-10}$ \\
540 & 1,64 & 11,4 \\
1380 & 1,17 & 8,2 \\
1980 & 1,24 & 8,6 \\
2880 & 1,17 & 8,2
\end{tabular}

Aus Plattenradius $(12,5 \mathrm{~cm})$ und Plattenabstand $(0,215 \mathrm{~cm})$ des Kondensators läBt sich seine Kapazität mit Luft als Dielektrikum berechnen. Man findet $C_{\mathrm{L}}=0,000202 \mathrm{MF}$, und mit Hilfe des aus Spannung und Stromstärke berechneten Widerstandes für die Leitfähigkeit: $\lambda=0,31 \cdot 10^{-14} \mathrm{~cm}^{-1} \Omega^{-1}$. 


\section{Kondensator F.}

Ioidener Flasche; $d=0,28 \mathrm{~cm}$.

a) Tabelle für den Nachladungsstrom.

(Schaltungsschema I.)

$$
\begin{aligned}
& E=103,2 \mathrm{~V} \quad G=0,300 \mathrm{MF} \quad W=10000 \Omega \\
& C_{0}=0,001145 \mathrm{MF} \\
& \vartheta=16,3^{\circ} \mathrm{C} . \quad \beta=0,116 \quad n=0,70
\end{aligned}
$$

\begin{tabular}{l|l|l|l|l|l|l|l|}
\hline$t \sec$ & $t^{\prime} \mathrm{sec}$ & $e$ coul & $J=i+j$ & $i$ & $n$ & $i_{\text {ber. }}$ & $\frac{\Delta C}{C_{0}}$ \\
\hline
\end{tabular}

Zeitmessung mit der Mikrometerschraube:

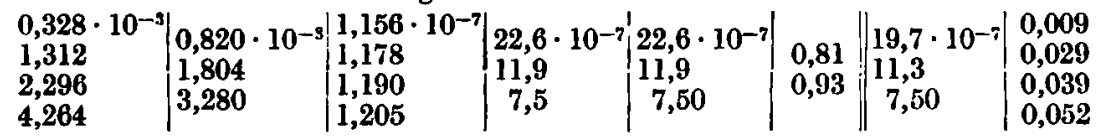

Zeitmessung mit dem Pendelquadranten:

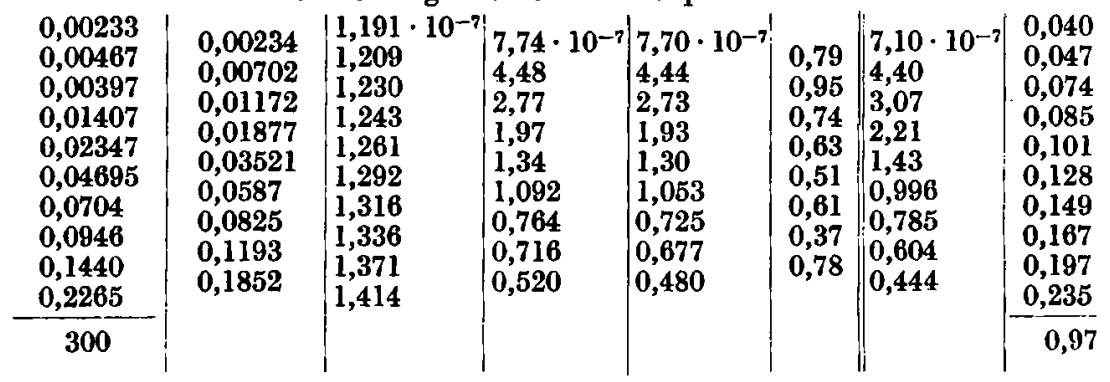

b) Tabelle für den Leitungsstrom.

(Schaltungssohema II.)

$$
\begin{aligned}
& E=103,2 \nabla \quad G=0,0100 \mathrm{MF} \quad \Delta t=0,1440 \mathrm{sec} \\
& \vartheta=16,3^{\circ} \mathrm{C} \text {. }
\end{aligned}
$$

\begin{tabular}{r|l|l}
\hline$t$ sec & c coul & $j$ \\
\hline 60 & $6,9 \cdot 10^{-10}$ & $48 \cdot 10^{-10}$ \\
180 & 6,1 & 42 \\
480 & 5,9 & 41 \\
1680 & 5,7 & 39 \\
1860 & 5,6 & 38 \\
2160 & 5,5 & 38 \\
2460 & 5,6 & 99
\end{tabular}

Wird die Dielektrizitätskonstante gleich 6,0 gewählt, so ergibt sich aus dieser Tabelle ein stationäres Leitvermögen $\mathrm{zu}: \lambda=1,75 \cdot 10^{-14} \mathrm{~cm}^{-1} \Omega^{-1}$. 
Die angeführten Beispiele von je drei Papier- und drei Glaskondensatoren, die ziemlich willkürlich gewählt waren, zeigen ein übereinstimmendes Verhalten, sowohl qualitativ wie quantitativ. Innerhalb der ersten Sekunde überwiegt der Nachladungsstrom den Leitungsstrom sehr stark; die gröBten Werte, die für den Nachladungsstrom noch gemessen wurden, sind mehr als das 500-1000fache des schlieBlich eintretenden stationären Leitungsstromes; bei den Papierkondensatoren $A$ und $B$ konnte ein solcher überhaupt nicht mehr sicher nachgewiesen werden. Innerhalb des gemessenen Zeitraumes von $0000328-0,2265$ Sek. läßt sich der Nachladungsstrom durch die Gleichung $i=E C_{0} \beta t^{-n}$ darstellen; eine Annäherung an einen bestimmten endlichen Grenzwert für sehr kleine Zeiten ist nicht zu erkennen. Das Zeitintervall nach noch kleineren Zeiten auszudehnen ist nicht möglich, weil man sonst in das Gebiet der ",normalen" Ladung des Kondensators kommt und dann veränderliche Potentialdifferenz an den Kondensatorbelegungen hat. Die Kondensatoren $C$ (Papier) und $F$ (Glas) weichen insofern ab, als sie das Potenzgesetz nicht so gut befolgen, immerhin dieses viel besser als z. B. ein Exponentialgesetz, wie man sich durch Rechnung leicht überzeugen kann; es darf auch in diesen Fällen zur näherungsweisen Darstellung benutzt werden. Die Materialkonstanten $\beta$ und $n$ sind bei den verschiedenen Beispielen an GröBe nicht sehr verschieden; sie seien hier kurz noch einmal zusammengestellt.

\begin{tabular}{c|c|c|c}
\hline \hline Kond. & Dielektrikum & $\beta$ & $n$ \\
\hline \hline$A$ & Papier & $\mathbf{0 , 0 5 1 1}$ & $\mathbf{0 , 7 8 9}$ \\
$B$ & $"$ & $\mathbf{0 , 1 5 8}$ & $\mathbf{0 , 7 4 7}$ \\
$C$ & $"$ & $\mathbf{0 , 2 2 6}$ & $\mathbf{0 , 7 8 5}$ \\
$D$ & Gles & $\mathbf{0 , 1 3 5}$ & $\mathbf{0 , 6 6 2}$ \\
$E$ & $n$ & $\mathbf{0 , 1 1 5}$ & $\mathbf{0 , 6 9 7}$ \\
$F$ & $"$ & $\mathbf{0 , 1 1 6}$ & $\mathbf{0 , 7 0 0}$
\end{tabular}

Es scheint sich hier um wirklich empirische Materialkonstanten zu handeln, die für die einzelnen Proben eines Materials nicht allru stark voneinander abweichen; es gilt diez besonders für den Exponenten n. Zum Vergleich seien 
noch die Werte aufgeführt, die E. $\nabla$. Schweidler ${ }^{1}$ ) aus galvanometrischen Beobachtungen des Entladungs- oder Rückstandstromes für Zeiten von 10 Sek. bis etwa 30 Min., also unter ganz anderen Bedingungen, erhielt; sie beziehen sich ebenfalls auf Zimmertemperatur.

\begin{tabular}{|c|c|c|}
\hline Dielektrikum & $\beta$ & $n$ \\
\hline Glas . . & 0,078 & 0,74 \\
\hline Paraffinpapier & 0,0154 & 0,70 \\
\hline
\end{tabular}

Ähnliche Werte entnimmt man den Kurven von H. Jordan ${ }^{2}$ ) ( $n=0,7$ für imprägnierte Papiere).

Aus den mit $\triangle C / C_{0}$ überschriebenen Kolonnen geht hervor, daß schätzungsweise die Hälfte der Rückstandsladung schon innerhalb der ersten Sekunde gebildet wird; dieses Verhalten ist charakteristisch für die Dielektriken Glas und imprägnierte Papiere. Einige Folgerungen für die Wagnersche Theorie der unvollkommenen Dielektriken sollen im Zusammenhang mit den Wechselstromverlusten besprochen werden.

b) Die Weohselstromverluste.

Diskussion der Resultate für feste Dielektriken.

Die Darstellung des Nachladungsstromes durch die Formel:

$$
\begin{gathered}
i=E C_{0} \varphi(t)=E C_{0} \beta t^{-n} ; \\
\varphi(t)=\beta t^{-n} ; \quad 0<n<1
\end{gathered}
$$

gestattet nun eine einfache Berechnung der Wechselstromverluste, die durch dielektrische Nachwirkung notwendig bedingt sind. Auch wenn außerhalb des Zeitraumes, für den die Formel empirisch bestimmt wurde, ihre Gültigkeit aufhören sollte, so erstreckt er sich doch weit genug, daß die Abweichungen für kleinere und größere Zeiten die Rechnungsresultate nicht mehr wesentlich beeinflussen können, wenigstens solange es sich nicht um Wechselströme sehr hoher Frequenz handelt. Der Effektverlust, der sich aus dem dielektrischen Verhalten ergibt, ist nach Formel (12) allgemein:

1) Vgl. E. v. Sohweidler, 1. c. p. 764 .

2) H. Jordan, l. c. 
dabei:

$$
Q=\omega C_{0} E_{\text {eff. }}^{2}\left(\frac{1}{\omega C_{0} I V}+B\right)
$$

$$
B=\int_{0}^{\infty} \varphi(u) \sin (\omega u) d u
$$

Für $\varphi(u)=\beta u^{-n}$ folgt:

$$
B=(\omega)^{n-1} \cdot \beta \cdot \frac{\pi}{2 \Gamma(n) \cos \frac{(1-n) \pi}{2}} .
$$

Wir berechnen den Effektverlust also nach dem Ausdruck:

$$
Q=\omega C_{0} E^{2} \text { eff. }\left\{\frac{1}{\omega C_{0} W}+\omega^{n-1} \cdot \beta \cdot \frac{\pi}{2 \Gamma(n) \cos \frac{(1-n) \pi}{2}}\right\} \text {. }
$$

Ein Vergleich mit der allgemeinen Beziehung:

$$
Q=\omega C_{0} E^{2}{ }_{\text {efr. }} \sin \delta,
$$

die für kleine Winkel $\delta$ gültig ist, zeigt, daß obige Klammer den Sinus des Verlustwinkels bedeutet. Von den Summanden der Klammer gibt der erste den Beitrag, den die Leitfähigkeit des Materiales an den Effektverlust liefert, der zweite den Beitrag der dielektrischen Nachwirkung. Wir wollen ersteren mit $\sin \delta_{1}$, den letzteren mit $\sin \delta_{2}$ bezeichnen und zunächst zeigen, daß $\sin \delta_{1} / \sin \delta_{2}$ so klein ist daB $\sin \delta_{1}$ neben $\sin \delta_{2}$ in der Rechnung vernachlässigt werden kann. Das würde heißen, daß der Effektverlust infolge dielektrischer Nachwirkung den Effektverlust durch Leitung bei weitem überwiegen muB, wie es auch durch das Überwiegen der anfänglichen Nachladungsströme über den stationären Leitungsstrom arschaulich ist. Diese Vergleichung ist in folgender Tabelle niedergelegt; berechnet für Wechselstrom von der Periodenzahl 50,3: •

\begin{tabular}{c|c|c|c|c}
\hline Kond. & Dielektrikum & $\sin \delta_{1}$ & $\sin \delta_{2}$ & $\frac{\sin \delta_{1}}{\sin \delta_{2}}$ \\
\hline$A$ & Papier & sehr klein & 0,0213 & $<0,001$ \\
$B$ & " & " & 0,0577 & $<0,001$ \\
$C$ & Glas & 0,000380 & 0,0900 & 0,0042 \\
$D$ &, & 0,000164 & 0,0260 & 0,0042 \\
$E$ &, & 0,000104 & 0,0276 & 0,0060 \\
$F$ & & & & 0,0038
\end{tabular}


Wie man sieht, bleibt $\sin \delta_{1}$ stets $100-1000 \mathrm{mal}$ kleiner als $\sin \delta_{2}$. Es ist zu beachten, daB mit steigender Frequenz dieses Verhältnis immer abnimmt.

Es ist daher die Rechnung unter Vernachlässigung von $\sin \delta_{1}$ gegen $\sin \delta_{2}$ durchgeführt worden. Die Resultate folgen in einer weiteren Tabelle, zusammengestellt mit den nach Schaltungsschema III mit dem Elektrodynamometer direkt gemessenen Werten der Hffektverluste. Der ebenfalls aufgeführte Sinus des Verlustwinkels ist aus dem gemessenen Effektverlust mit Hilfe der bekannten Kapazität, Frequenz und Effektivspannung berechnet. Die Meßgenauigkeit beträgt etwa 3-4 Proz.; berücksichtigt man noch etwaige unkontrollierte Temperaturschwankungen, sowie mögliche Einflüsse wechselnder Luftfeuchtigkeit, so wird man mit einem Fehler von rund 5 Proz. qu rechnen haben. Die Messungen des Nachladungsstromes nach Schaltungsschema I und II und die Effektmessung nach Schaltungsschema III wurden für einen bestimmten Kondensator stets am gleichen Tage vorgenommen.

Tabelle der Fffektverluste für feste Dielektriken.

$$
\begin{aligned}
E & \multicolumn{1}{c}{\text { Bezeichnungen: }} \\
\omega=2 \pi \nu & \text { Erektivepannung des Wechselstromes }(V), \\
\nu & \text { Periodequenz, } \\
\varepsilon & \text { Ernpfindlichl pro Sekunde, } \\
& \text { in Erg/sec pro Skalekteil, } \\
Q & \text { Effektverlust in Erg/sec, } \\
\delta & \text { Verlustwinkel, }
\end{aligned}
$$

\begin{tabular}{|c|c|c|c|c|c|c|c|c|}
\hline Kond. & Dielek. & $E(\mathbf{V})$ & y & $\varepsilon$ & $\sin \delta$ & $Q_{\text {gem. }}$ & $Q$ wer. & $\frac{\Delta Q}{Q} \%$ \\
\hline$A$ & Papier & 105,3 & 50,3 & 8,88 & 0,0260 & 835 & 867 & $+3,8$ \\
\hline$B$ & ", & 105,4 & 50,3 & 7,94 & 0,0555 & 3390 & 3533 & $+4,1$ \\
\hline$C$ & , & 104,5 & 50,3 & 24,4 & 0,0903 & 11390 & 11340 & $-0,4$ \\
\hline$D$ & Glas & 105,2 & 50,3 & 11,60 & 0,0256 & 3750 & 3810 & $+1,6$ \\
\hline$E$ & ", & 104,8 & 50,3 & 7,85 & 0,0218 & 1263 & 1450 & $+13,8$ \\
\hline$F$ & ", & 105,3 & 50,3 & 7,80 & 0,0218 & 1108 & 1360 & +23 \\
\hline
\end{tabular}

$\Delta Q / Q$ Proz. Abweichung von Rechnung und Messung in Proz.

Diese Tabelle enthält die Hauptresultate für die festen Dielektriken. Aus ihr geht hervor, daß sich aus den dielektrischen Nachwirkungserscheinungen statischer Natur die 
Wechselstromverluste für nicht hohe Frequenzen und Spannungen berechnen lassen; zum T'eil liegt die Übereinstimmung ganz innerhalb der Fehlergrenzen der Messung, zum Teil ist die qualitative Übereinstimmung wenigstens nachgewiesen. Es gelang mir nicht, den Grund der Abweichungen, den der zweitletzte und namentlich der letzto Glaskondensator zeigen, sicher festzustellen; diese Differenzen ergaben sich auch bei wiederholten Messungen. Man kann vermuten, daß ein anomaler Ieitungseffekt, ähnlich wie wir ihn bei den flüssigen Dielektriken werden kennen lernen, die Messung des Nachladungsstromes fälscht, oder da $\beta$ diese Dielektriken, bei denen ja ein stationärer Leitungsstrom nachgewiesen werden konnte, die Toraussetzung der Theorie, Umkehrbarkeit der Ladungsrorgänge (Superpositionsprinzip) nicht streng erfüllen. Bessere Übereinstmmung zwischen Beobachtung und Berechnung zeigen die Papierkondensatoren; auf sie ist die Theorie offenbar in vollem Umfange anwendbar. Sie erwiesen sich auch, mit Ausnahme des dritten $(C)$, als bedeutend bessere Isolatoren als Glas. Aus dem Ganzen geht jedoch deutlich bervor, daß die Wechselstromverluste vor allem der dielektrischen Nachwirkung zugeschrieben werden müssen; die Leitungsverluste durch Joulesche Wärme kommen dagegen gar nicht in Betracht. Es wird dies bei hohen Frequenzen noch mehr der Fall sein, als bei niederen.

Wir haben die Berechnung mit Hilfe der empirischen Näherungsformel $\varphi(t)=\beta t^{-n}, 0<n<1$, durchgeführt; infolgedessen können in der Rechnung noch Fehler stecken, die vom Gültigkeitsbereich dieser. Näherungsformel abhängen. Da für $t=0, \varphi(t)=\infty$ wird, ist möglicherweise ein zu großer berechneter Effektverlust zu erwarten. Auf das physikalisch Unwahrscheinliche eines unendlich großen Anfangwertes für $\varphi(t)$ wurde schon p. 311 bingewiesen; es würde dies einen unendlich großen Anfangswert des Nachladungsstromes bedingen. Auch gibt die vollständige Wagnersche Theorie einen endlichen Betrag für den Beginn des Nachladungsstromes. Offenbar ist dieser Betrag bei unseren Dielektriken aber verhälnismäBig sehr groß, zeigte doch die Messung des Nachladungsstromes selbst bei sehr kleinen Zeiten von ungefähr 0,001 Sek. keine Annäherung an eine endliche Grenze. Übrigens würde man praktisch, auch wenn man der Näherungsformel 


$$
\varphi(t)=\beta^{-n}, \quad 0<n \ldots .
$$

unbeschränkte Gültigkeit zuspräche, nie zu unendlich großen Nachladungsströmen geführt, so wenig als praktisch unendlich groBe Leitungsströme vorkommen. Denn da der Nachladungsstrom der Potentialdifferenz an den Kondensatorbelegungen proportional ist, ein Kondensator sich aber infolge der Zuleitungswiderstände immer erst in endlicher Zeit auf das erteilte Potential aufladet, so folgt als genaue Formel für den Nachladungsstrom:

$$
i=E\left(1-e^{-\frac{1}{W c_{0}} t}\right) \beta C_{0} t^{-n} \text {. }
$$

Dieser Ausdruck verschwindet für $t=0$. (i $)_{t=0}=0$. Analog beginnt bei sinustörmiger Spannung der Nachladungsstrom mit dem Werte Null:

$$
\begin{gathered}
i=E_{0} \sin \omega t \beta C_{0} t^{-n} \\
(i)_{t=0}=0 .
\end{gathered}
$$

Damit soll nicht versucht werden, die Form $\varphi(t)=\beta t^{-n}$ über ihre empirische Bedeutung hinauszuheben; es wird aber verständlich gemacht, $\mathrm{da} B$ es praktisch unmöglich sein kann, den wahren Anfangswert von $\varphi(t)$ zu finden; er wird eben durch die „normalen" Ladungsvorgänge des Kondensators, schließlich vollständig verwischt. Zur Veranschaulichung seien zwei Beispiele angeführt, bei denen ich mit der Zeitmessung mit der Mikrometerschraube (Schaltungsschema I) so weit hinunter ging, daß noch die ganze ,normale" Ladung des Versuchskondensators bestimmt wurde. Durch Bildung der Differenzenquotienten nach der Zeit erhielt man wieder dio Gesamtstromstärke $J$; sie setzt sich zusammen aus der Stromstärke des ,normalen" Ladungsstromes $i^{\prime}$ und des Nachladungsstromes $i$. Erstere kann man berechnen aus der elektromotorischen Kraft $E$, dem Widerstand $W$ und der Kapazität $C_{0}$, letztere aus den bekannten Materialkonstanten und der Formel

$$
i=E\left(1-e^{-\frac{1}{W C_{0}}}\right) \beta C_{0} t^{-n},
$$

so daß ein zahlenmäBiger Vergleich möglich ist. Also:

$$
\left\{\begin{array}{l}
J=i^{\prime}+i \\
i^{\prime}=\frac{E}{W} e^{-\frac{1}{W C_{0}} t} \\
i=E\left(1-e^{\left.-\frac{1}{W C_{0}}\right)} \beta C_{0} t^{-n}\right.
\end{array}\right.
$$


Dielektrische Effektcerluste von Kondensatoren usw. $\mathbf{3 4 7}$

Kondensator B (Papier).

\begin{tabular}{c|c|c|c|c}
$E=103,9 \vee$ & $\begin{array}{c}C_{0}=0,001740 \mathrm{NFF} \\
\beta=0,158\end{array}$ & $\begin{array}{c}G=0,502 \mathrm{MF} \\
n=0,75\end{array}$ & $W=10000 \Omega$ \\
\hline$t$ sec & ${ }^{{ }_{\text {ber. }}}$ & $i_{\text {ber. }}^{\prime}$ & $J_{\text {ber. }}$ & $J_{\text {gem. }}$ \\
\hline $1,64 \cdot 10^{-6}$ & $598 \cdot 10^{-7}$ & $94270 \cdot 10^{-7}$ & $94870 \cdot 10^{-7}$ & $90450 \cdot 10^{-7}$ \\
4,92 & 731 & 78050 & 78781 & 84750 \\
8,20 & 750 & 65000 & 65750 & 52100 \\
14,78 & 746 & 44350 & 45100 & 47770 \\
34,44 & 596 & 14330 & 14930 & 13470 \\
50,92 & 488 & 5580 & 6070 & 6940 \\
223,4 & 170 & 0,29 & 170,5 & 200
\end{tabular}

Kondensator D (Glas).

$$
\begin{array}{rlrl}
E=103 ; 8 \mathrm{~V} & C_{0}=0,00419 \mathrm{MF} & G=1,002 \mathrm{MF} & W=10000 \Omega \\
\beta & =0,135 & n=0,66 &
\end{array}
$$

\begin{tabular}{c|l|l|l|l}
\hline \multicolumn{1}{c|}{$i_{\text {sec }}$} & $i_{\text {ber. }}$ & \multicolumn{1}{c|}{$i_{\text {ber. }}^{\prime}$} & $J_{\text {ber. }}$ & $J_{\text {gem. }}$ \\
\hline $1,64 \cdot 10^{-6}$ & $152 \cdot 10^{-7}$ & $99420 \cdot 10^{-7}$ & $99570 \cdot 10^{-7}$ & $113200 \cdot 10^{-7}$ \\
4,93 & 212 & 91880 & 92090 & 94500 \\
8,20 & 243 & 85000 & 85250 & 82300 \\
11,49 & 260 & 78580 & 78840 & 76100 \\
21,38 & 282 & 63120 & 63400 & 57200 \\
37,80 & 296 & 4200 & 42300 & 43300
\end{tabular}

Die Messung so kleiner Zeiten geht an die Leistungsgrenze des Pendels. Man sieht aber, daB der normale Ladungsstrom den Nachladungsstrom vollkommen überdeckt, so daß aus dem gemessenen Strom $J_{\text {gem. }}$ über letzteren nichts mehr ausgesagt werden kann.

Aus all dem geht hervor, daß die Formel

$$
\varphi(t)=\beta t^{-n}
$$

für die Nachwirkungsfunktion $\varphi(t)$ als gute Näherung mit großem Gültigkeitsbereich zu betrachten ist. ${ }^{1}$ ) Sio ergibt sich aus der Wagnerschen Theorie der unvollkommenen Dielektriken bei kleinen Verteilungskonstanten ,b". Es ist dann (vgl. p. 814):

$$
n=1+2 b^{2} \log \text { nat } \frac{\tau}{T_{0}}
$$

1) Vgl. auch das über die Messungen von H. Jordan gesagte, K. W. Wagner, l. c. 
wo $\tau$ den Zeitwert darstellt, in dessen Ungebung diese Beziehung gilt. Für den Verlustwinkel besteht im Grenzfalle eines sehr kleinen , $b$ " ferner die einfache Beziehung:

$$
\lim _{b=0}\left(1+\frac{\Delta C}{C^{-}}\right) \operatorname{tg} \delta=\frac{\sqrt{\pi}}{2} k b=0,886 k b .
$$

Ninmt man noch hinzu, daB für

$$
\omega=0, \quad \frac{\Delta C}{C_{0}}=\frac{\Delta C_{\infty}}{C_{0}}=k
$$

ist ${ }^{1}$ ), so lassen sich aus den experimentellen Daten die Größen $k, b$ und $T_{0}$ schätzungsweise bestimmen. Unter Vernachlässigung von $\triangle C ; C_{0}$ gegen 1 , was für Wechselstrom von 50 Perioden auf einen Fehler von höchstens einigen Prozenten hinauskommt, und unter Gleichsetzung von $\operatorname{tg} \delta=\sin \delta$, da ja $\delta$ ein kleiner Winkel ist, berechnet man:

\begin{tabular}{c|c|c|c|c}
\hline Kond. & $1-n$ & $\sin \delta$ & $k=\frac{\Delta C_{\infty}}{C_{0}}$ & $b$ \\
\hline$A$ & 0,211 & 0,0260 & 0,75 & 0,039 \\
$B$ & 0,253 & 0,0555 & 1,38 & 0,046 \\
$C$ & 0,215 & 0,0903 & 1,53 & 0,067 \\
$D$ & 0,338 & 0,0256 & 0,83 & 0,035 \\
$F$ & 0,303 & 0,0218 & 0,78 & 0,032 \\
$F$ & 0,300 & 0,0218 & 0,97 & 0,025
\end{tabular}

Man erhält in der Tat eine kleine Verteilungskonstante $b .{ }^{2}$ ) Dies hat eine äußerst lose Gruppierung der verschiedenen Zeitkonstanten $T$ urn ihren wahrscheinlichsten Wert $T_{0}$ zur Folge. Dieser selbst berechnet sich zu einer hohen Zehnerpotenz, so daß die ihm entsprechende Nachwirkung sich z. B. noch nach Jabren bemerkbar machen muß. Nachwirkungen äbnlich großer Dauer sind allerdings schon beobachtet worden. ${ }^{3}$ )

1) K. W. Wagner, l. c. p. 841 .

2) Wir möchten nicht versäumen, darauf hinzuweisen, daß Beobachtungen über die elastische. Nachwirkung von Glas zu Konstanten analoger Größe führen. Vgl. E. Wiechert, l. c. p. 549.

3) E. v. Sobweidler, l. c. p. 758; auch E. Schreiber, Ann. d. Phys. p. 806. 1913. 


\section{Flüissige Dlelektriken.}

a) Die Anomalien des Leitungastromes.

Die flüssigen Dielektriken wurden in vernickelten Zylinderkondensatoren untersucht, die vom Mechaniker des Institutes hergestellt waren. Zwei Röhrensysteme $a, a, a$ und $b, b, b$ sind je auf eine gestufte Scheibe $A$ bzw. $B$ mittels fein geschnittener Gewinde aufgeschraubt. Das System $a, a, a$ hängt in der Deckelscheibe $D$ aus $0,5 \mathrm{~cm}$ dickem Spiegelglas. Durch den Ring $R$, über den die Deckelscheibe etwas vorsteht, wird es in den äußersten Zylinder des Systemes $b, b, b$ geklemmt und festgehalten, so da.B die beiden Zylindersysteme genau konzentrisch ineinander passen. Die Deckelscheibe besitzt ein Loch zum Einfüllen der Flüssigkeit bzw. zum Eintauchen eines Thermometers. Die inneren Zylinder sind mit kleinen

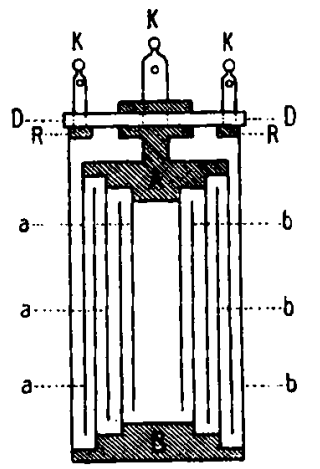

Fig. 4. Durchbohrungen versehen, zum Ausgleich der Flüssigkeit bei ungleicher Füllung. $K, K$ sind die Anschlußklemmen. Di Kondensatoren konnten vollständig zerlegt und nach Gebrauch die Bestandteile einzeln gereinigt werden. Sie standen mir in drei verschiedenen Größen zur Verfügung:

$\begin{array}{ccccc}\text { Größe } & \text { Höhe } & \text { Durchmesser } & \text { Zahl der Zylinder } & \text { Kapazität (Luft) } \\ A & 160 \mathrm{~mm} & \mathbf{5 5} \mathrm{mm} & 4+4 & 4,20 \cdot 10^{-4} \mathrm{MF} \\ B & 160, " & 47, & 3+3 & 1,675 \cdot 10^{-4} ", \\ C & 200 ", & 60 " & 2+2 & 0,960 \cdot 10^{-4} ",\end{array}$

Das innere Zylindersystem kam mit der Spannungsquelle in Verbindung; an das äußere wurde der Hilliskondensator bzw. das Elektrodynamometer angeschlossen. Die Zuverlässigkeit der Isolation der einzelnen Leitungsteile wurde von Zeit $\mathrm{zu}$ 'Leit mit dem Blattelektroskop nachgeprüft.

Im Laufe der Untersuchung zeigte es sich, daß die flüssigen Dielektriken einige Analogien, aber auch prinzipielle Unterschiede zum Verhalten fester Dielektriken bei Gleicbstrom und bei Wechselstrom aufweisen.

Beim Anlegen einer lronstanten Spannung an einen Flüssigkeitskondensator tritt ein Strom auf, der in einem großen 
Zeitintervall übereinstimmenden Verlauf hat wie der Nachladungsstrom fester Dielektriken ${ }^{\mathbf{1}}$ ); es gilt für ihn auch das Gesetz:

$$
i=B t^{-n} ; \quad 0<n<1 .
$$

Nach dem Beispiele früherer Beobachter prüfte ich dies mit einer einfachen Messung mit Hilfe eines empfindlichen Galvanometers. Die Schaltung ist beistehend skizziert:

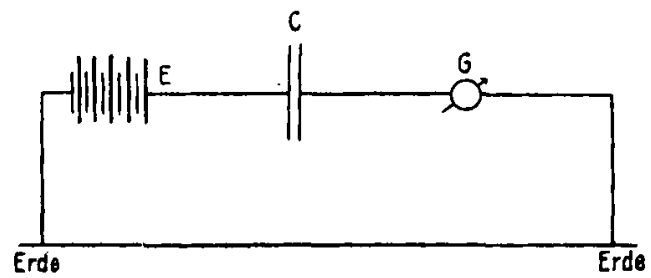

Fig. 5.

$E$ bedeutet eine Hochspannungsbatterie (KlingelfuB) von rund 125 Volt, $C$ den Versuchskondensator, $G$ das Galvanometer. Letzteres, ein Instrument von Siemens \& Halske, eichte ich absolut durch Stromabzweigung und fand bei einem Skalenabstand von $2300 \mathrm{~mm}$ eine Empfindlichkeit von 2,27 $\cdot 10^{-10} A$ pro Skalenteil. Auf diese Weise ergaben sich z. B. für Schwefelkohlenstoff und Petroleum innerhalb eines Zeitraumes von 1-30 Minuten folgende Resultate:

Kondensa tor B (Schwefelkohlenstoff).

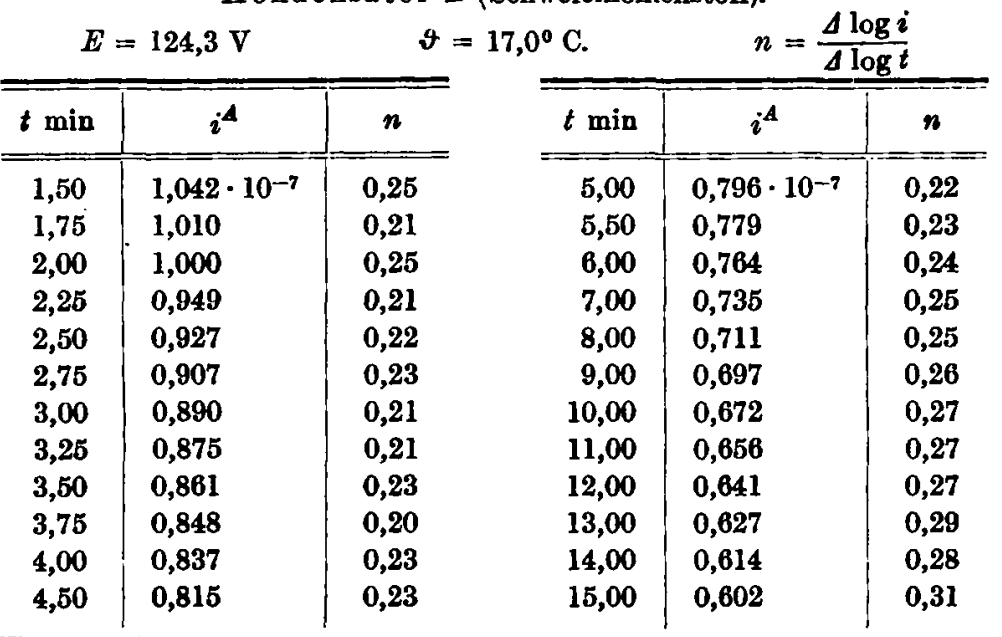

1) Vgl. z. B. auoh E. v. Sohweidler, l. c. p. 754. 
Kondensator A (Petroleum).

$E=125,2 \mathrm{~V}$

\begin{tabular}{c|l|c}
\hline \hline$t \min$ & \multicolumn{1}{|c|}{$i^{\Lambda}$} & $*$ \\
\hline \hline 1,50 & $438,8 \cdot 10^{-10}$ & 0,42 \\
1,75 & 412,7 & 0,39 \\
2,00 & 390,8 & 0,43 \\
2,25 & 371,6 & 0,39 \\
2,50 & 356,8 & 0,37 \\
2,75 & 344,3 & 0,32 \\
3,00 & 334,8 & 0,37 \\
4,00 & 300,8 & 0,39 \\
5,00 & 276,0 & 0,42
\end{tabular}

$\vartheta=16,5^{\circ} \mathrm{C}$.

\begin{tabular}{r|l|c}
\hline \hline$t \min$ & \multicolumn{1}{|c|}{$i^{A}$} & \multicolumn{1}{|c}{$n$} \\
\hline \hline 6,00 & $255,8 \cdot 10^{-10}$ & 0,45 \\
7,00 & 238,6 & 0,54 \\
8,00 & 222,0 & 0,48 \\
9,00 & 209,0 & 0,64 \\
10,00 & 195,9 & 0,29 \\
15,00 & 154,4 & 0,22 \\
20,00 & 128,9 & 0,18 \\
25,00 & 122,1 & 0,18 \\
30,00 & 118,1 & -
\end{tabular}

Die Übereinstimmung des Stromverlaufes in.erster Näherung mit dem Gesetze $i=B t^{-n}$ ist unverkennbar, wenn auch beim Schwefelkohlenstoff der Exponent $n$ mit wachsender Zeit langsam zunimmt; beim Petroleum dagegen sind die letzten Exponenten kleiner als die ersten. Dieser Strom ist trotz seiner Ähnlichkeit mit dem Nachladungsstrom fester Dielektriken scharf ron jenem auseinander zu halten. ${ }^{1}$ ) Vor allem ist er nicht umkehrbar, d. h. er gibt zu keiner Rüickstandsbildung Veranlassung. Ich konnte bei allen Flüissigkeiten, die ich untersuchte, sowohl mit dem Galvanometer als mit dem Elektrometer stets nur Spuren von Rückstand oder chemischer Polarisation der Elektroden finden, die nach einigen Minuten Kurzschlusses verschwunden waren.

Man hat bei den Flüssigkeiten ohne Zweifel eigentliche Ionenleitung an\%unehmen. $\mathrm{Ob}$ diese einer bestimmten Eigenleitfähigkeit der Substanz zukommt, erscheint jedoch fraglich, da sie von zufälligen, oft spurenhaften Verunreinigungen stark abhängt. Es liegt eher nahe, wenigstens bei unseren Beispielen, diesen Verunreinigungen den Grund der Leitung zuzuschreiben und das ideal reine Dielektrikum als vollkommenen Nichtleiter anzusehen.

Wio aus den Untersuchungen von $H$. Jordan ${ }^{2}$ ) und auch aus unseren Resultaten hervorgeht, ist bei einigen festen

1) Vgl. E. v. Schweidler, 1. c. p. 755.

2) H. Jordan, l. o. 
Dielektriken der Gültigkeitsbereich der Formel für den Nachladungsstrom $i=B t^{-n}$ ein großer; man kann aus dem Verlauf bei späteren. Zeiten auf den Verlauf bei sehr kleinen Zeiten und auf die Wechselstromeffektverluste zurückschließen. Flüssigkeiten zeigen hier ein ganz abweichendes Verhalten. Vor der Messung nit dem Galvanometer bestimmte ich nach Schaltungsschema I die Stromstärken, die ganz zu Beginn in einem Zeitraume von 0,005-0,225 Sek. auftreten. Diese verglich ich mit; den entsprechenden Stromstärken, die man aus den Galvanonetermessungen extrapoliert. Die Zahlen seien für die aufgeführten Beispiele des Kondensators mit Schwefelkohleustoff und nit Petroleum mitgeteilt:

\begin{tabular}{|c|c|c|c|c|}
\hline \multirow{3}{*}{$E=124,3$} & \multicolumn{3}{|c|}{$\begin{array}{c}\text { Kondensa tor B (Schwefelkohlenstoff). } \\
\text { Anfangsstromstärken: }\end{array}$} & \multirow[b]{2}{*}{$\vartheta=17,0^{\circ} \mathrm{C}$} \\
\hline & $G=0$ & MF $\quad W$ & $10000 \Omega$ & \\
\hline & \multicolumn{4}{|c|}{ Extrapolationsformel: $i=2,86 \cdot t^{-0,225} \cdot 10^{-7} \mathrm{~A} / \mathrm{sec}$} \\
\hline$t \mathrm{sec}$ & $t^{\prime}$ sec & $e$ coul & $i^{A}$ & $i^{A}$ ber. \\
\hline 0,00467 & & $0,0541 \cdot 10^{-0}$ & & $9,60 \cdot 10^{-7}$ \\
\hline 0,1183 & $\begin{array}{l}0,0825 \\
0,1724\end{array}$ & 0,1048 & $\begin{array}{l}4,46 \cdot 10^{-r} \\
4,46\end{array}$ & 7,79 \\
\hline 0,2265 & & 0,1531 & & 6,73 \\
\hline
\end{tabular}

Kondensator A (Petroleum).

Anfangsstromstärken:

$E=125,2 \mathrm{~V} \quad Q=0,300 \mathrm{MF} \quad W=10000 \Omega \quad \vartheta=16,9^{\circ} \mathrm{C}$.

Extrapolationsformel: $i=6,13 \cdot t^{-0,394} \cdot 10^{-7} 1 / \mathrm{sec}$.

\begin{tabular}{c|c|l|l|l}
\hline \multicolumn{1}{c|}{$t$ sec } & $t^{\prime}$ sec & \multicolumn{1}{c}{ e coul } & \multicolumn{1}{|c|}{$i^{A}$} & \multicolumn{1}{|c}{$i^{A}$ ber. } \\
\hline \hline 0,00467 & 0,0141 & $1,078 \cdot 10^{-7}$ & $0,63 \cdot 10^{-7}$ & $32,9 \cdot 10^{-7}$ \\
0,0235 & 0,0470 & 1,090 & 0,48 & 20,5 \\
0,0704 & 0,0478 & 1,113 & 0,63 & 15,6 \\
0,1183 & 0,0515 & 1,143 & 0,62 & 13,2 \\
0,1698 & 0,0567 & 1,175 & 0,66 & 11,6 \\
0,2265 & & 1,213 & &
\end{tabular}

Von einer Übereinstimmung zwischen Messung und Rechnung ist keine Rede; vielmehr zeigt sich die bemerkenswerte 'Tatsache, daß bei flüssigen Dielektriken die Stromstärke mit konstanten, verhältnismäßig nicht sehr großen Werten beginnt. Man hat also einen zeitlichen Verlauf folgender Art anzunehmen: 
Dielektrische Effektverluste von Kondensatoren usw. $\quad \mathbf{3 5 3}$

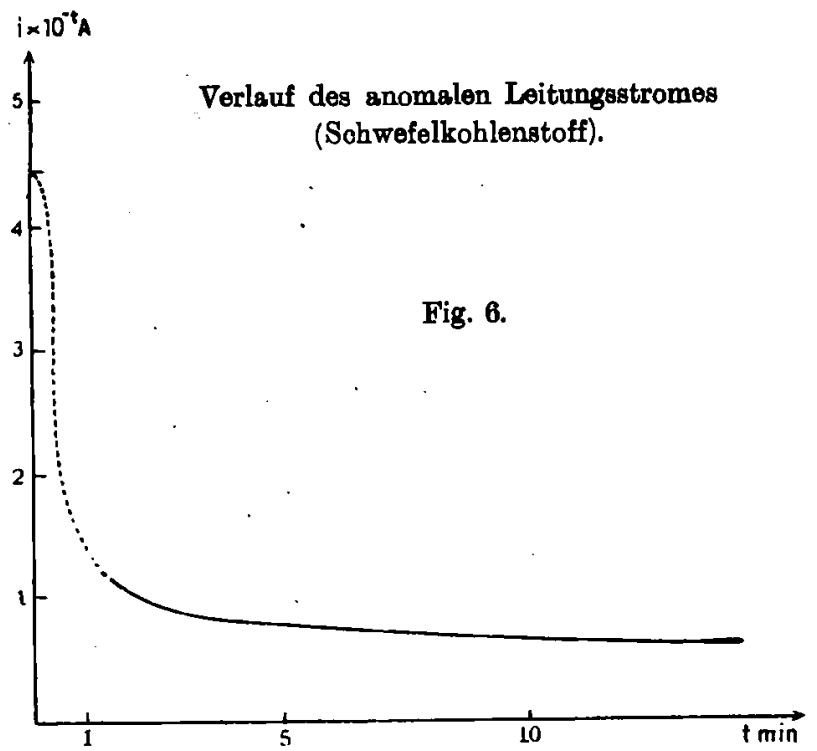

Das punktierte Stück der Kurve ist nichi beobachtet. Für die Berechnung der Weohselstrometfektverluste folgt, daB diese der konstanten Anfangsleitfähigkeit entsprechen müssen und daher nicht von der Frequenz abbängen werden.

b) Die Wechselstromverluste.

Es wurde an einer Reihe flüssiger Dielektriken nach den ausführlich beschriebenen Methoden die Anfangsleitfähigkeit. bzw. der Anfangswiderstand gemessen (Schaltungsschema I). Daraus war der Wechselstromverlust zu berechnen, der am Elektrodynamometer zum Vergleich direkt bestimmt wurde (Schaltungsschema III). Die Ergebnisse sind folgende:

1. Schroefelkohlenstoff.

a) Gleichstrom.

\begin{tabular}{|c|c|c|c|}
\hline$E=103,7$ & $\theta=$ & $02 \mathrm{MF}$ & $\vartheta=16,8^{\circ} \mathrm{C}$ \\
\hline$t \mathrm{sec}$ & $e$ coul & $i^{A}$ & $\bar{W}^{\Omega}$ \\
\hline $\begin{array}{l}0,00233 \\
0,0235 \\
0,0470 \\
0,0704 \\
0,0946 \\
0,1183 \\
0,1440 \\
0,1698 \\
0,1973\end{array}$ & $\begin{array}{l}1,138 \cdot 10^{-7} \\
1,440 \\
1,776 \\
2,125 \\
2,475 \\
2,848 \\
3,228 \\
3,642 \\
4,082\end{array}$ & $\begin{array}{l}14,1 \cdot 10^{-7} \\
13,3 \\
14,9 \\
14,5 \\
15,1 \\
14,7 \\
16,1 \\
15,9\end{array}$ & $\begin{array}{l}7,31 \cdot 10^{-7} \\
7,75 \\
6,93 \\
7,12 \\
6,84 \\
7,02 \\
6,40 \\
6,50\end{array}$ \\
\hline
\end{tabular}

Annalen der Phyaik. IV. Folge. 48. 
b) Wechselstrom.

$$
\begin{aligned}
E & =105,3 \mathrm{~V} & \nu & =50,4 \\
Q_{\text {gem. }} & =1529 \mathrm{Erg} / \mathrm{sec} & Q_{\text {ber. }} & =1520 \mathrm{Erg} / \mathrm{sec}
\end{aligned}
$$

2. Terpentinöl.

a) Gleichstrom.

$$
\begin{aligned}
& E=103,8 \mathrm{~V} \quad \theta=1,002 \mathrm{MF} \quad \vartheta=16,5^{\circ} \mathrm{C} \text {. } \\
& \begin{array}{l|l|l}
\hline \hline t \text { sec } & \multicolumn{1}{|c|}{e \text { coul }} & \multicolumn{1}{|c}{i^{4}} \\
\hline \hline 0,00233 & 1,370 \cdot 10^{-7} & 67,6 \cdot 10^{-7} \\
0,0235 & 2,819 & 68,7 \\
0,0470 & 4,428 & 70,3 \\
0,0704 & 6,095 & 70,1 \\
0,0946 & 7,755 &
\end{array} \\
& E=105,3 \mathrm{Y} \\
& Q_{\text {gem. }}=7150 \mathrm{Erg} / \mathrm{sec} \\
& \nu=50,3 \\
& Q_{\text {ber. }}=7180 \mathrm{Erg} / \mathrm{sec}
\end{aligned}
$$

3. Rizinusöl.

a) Gleichstrom.

$$
\begin{aligned}
& E=103,5 \mathrm{~V} \quad G=0,502 \mathrm{MF} \quad \vartheta=16,5^{\circ} \mathrm{C} \text {. } \\
& \begin{array}{l|l|l}
\hline \multicolumn{1}{c|}{t \text { sec }} & \multicolumn{1}{|c}{\text { coul }} & \multicolumn{1}{|c}{i^{\Lambda}} \\
\hline \hline 0,00283 & 1,985 \cdot 10^{-7} & 2,86 \cdot 10^{-7} \\
0,0235 & 2,044 & 2,88 \\
0,0704 & 2,177 & 2,80 \\
0,1183 & 2,312 & 2,76 \\
0,1698 & 2,451 & 2,79 \\
0,2265 & 2,612 &
\end{array} \\
& E=105,1 \mathrm{~V} \\
& Q_{\text {gem. }}=308 \mathrm{Erg} / \mathrm{sec} \\
& \begin{aligned}
\nu & =50,3 \\
Q_{\text {ber. }} & =285,5 \mathrm{Erg} / \mathrm{sec}
\end{aligned}
\end{aligned}
$$

4. Bonxol (mehrmals gebraucht).

a) Gleiohstrom.

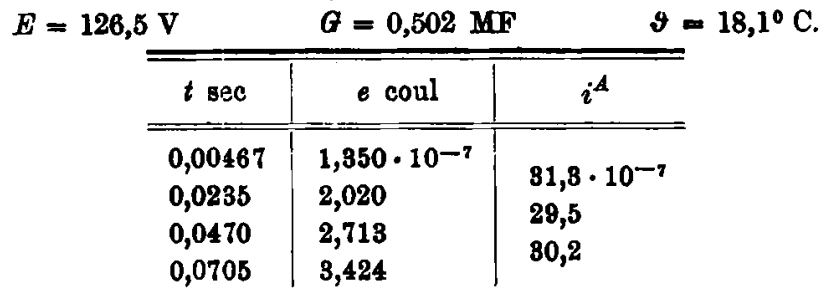


b) Wechselstrom.

$$
\begin{aligned}
E & =105,2 \mathrm{~V} & \nu & =50,2 \\
Q_{\text {gem. }} & =2580 \mathrm{Erg} / \mathrm{sec} & Q_{\text {ber. }} & =2500 \mathrm{Erg} / \mathrm{sec}
\end{aligned}
$$

\begin{tabular}{|c|c|c|}
\hline$E=125,4 \mathrm{~V}$ & $G=0,200 \mathrm{I}$ & $\vartheta=17,8^{\circ} \mathrm{C}$. \\
\hline$t$ sec & $c$ coul & $i^{A}$ \\
\hline 0,0234 & $1,144 \cdot 10^{-7}$ & \\
\hline 0,0705 & 1,206 & $1,82 \cdot 10^{-7}$ \\
\hline 0,1183 & 1,269 & 1,32 \\
\hline 0,1698 & 1,381 & 1,20 \\
\hline 0,2265 & 1,379 & 1,20 \\
\hline
\end{tabular}

\section{Petroleum.}

a) Gleichstrom.

b) Wechselstrom.

$$
\begin{aligned}
E & =104,7 \mathrm{~V} & \nu & =50,3 \\
Q_{\text {gem. }} & =112 \mathrm{Erg} / \mathrm{sec} & Q_{\text {ber. }} & =105,0 \mathrm{Erg} / \mathrm{sec}
\end{aligned}
$$

Aus den angeführten Beispielen geht die Konstanz der Anfangsleitwerte sowie die Übereinstimmung der daraus berechneter Effektverluste mit den direkt gemessenen deutlich hervor. Es ist somit, im Gegensatz zu den festen Dielekiriken, keine Abhängigkeit dieser Verluste von der Frequenz zu erwarten. Dieser Umstand, der in engstem Zusammenhange damit steht, daß feste Dielektriken Rückstände bilden, flüssige aber keine, bedeutet den fundamentalen Unterschied der festen und flüssigen Dielektriken bezüglich der Effektverluste im elektrischen Wechselfeld.

Die Verluste flüssiger Dielektriken sind eigentlicher Leitfähigkeit zuzuschreiben. Sie ist jedoch nicht als eine für die Substanz charakteristische Größe anzusehen, sondern wechselt mit deren Reinheit und besonderer Beschaffenheit sehr stark. Bei Paraffinöl und Vaselinöl, die als gute Isolatoren bekannt sind, lieB sich kein meBbarer Effektverlust nachweisen; ebenso bei ganz reinem Petroleum, Benzol (Kahlbaum), Xylol (Kahlbaum) und Toluol (Kahlbaum). Bei Schwefelkohlenstoff, mit dem ich ausführliche Versuche unternahm, konnten durch wiederholte Destillation Leitfähigkeit und Wechselstromverlust bis auf den zehnten Teil des ursprünglichen Wertes heruntergedrückt werden. Die mitgeteilten Resultate beziehen 
sich auf käufliche Substanzen, die weiter keinem Reinigungsproze $B$ unterworfen wurden. $\mathrm{Da} B$ bei flüssigen Dielektriken, die ein äußerst kleines Leitvermögen besitzen, fast keine Wechselstromverluste nachzuweisen sind, ist schon von früheren Reobachtern festgestellt worden. ${ }^{1}$ )

Es mag beiläufig noch erwähnt werden, daß die zur $\mathrm{Be}-$ stimmung der Leitfähigkeit flüssiger Dielektriken benutzte Methode (Schaltungsschema I) zugleich zu einer bequemen Bestinmung der Dielektrizitätskonstanten dienen kann. Man hat die Größe e, die Elektrizitätsmenge, die durch die Zuleitung des Kondensators geflossen ist, linear auf die Zeit Null zu extrapolieren, und erhält dann als Kapaxität des mit Flüssigkeit gefüllten Kondensators:

$$
C_{\mathrm{F}}=\frac{e_{0}}{E}
$$

Da die Kapazität mit Luft als Zwischenmedium bekannt ist, so ergibt sich:

$$
\varepsilon=\frac{C_{\mathrm{F}}}{C_{\mathrm{L}}}
$$

Für Schwefelkohlenstoff lindet man z. B. aus dem Beispiel 1:

$$
\varepsilon=\frac{1,069 \cdot 10^{-9}}{0,420 \cdot 10^{-9}}=2,55 \text {. }
$$

Bohlus.

Es war das Ziel der vorliegenden Untẹrsuchung, die Effektverluste von Kondensatoren im elektrischen Wechselfelde direkt zu messen und zu versuchen, ob sie sich quantitativ mit den Anomalien der Isadung und der Leitfähigkeit in Zusammenhang bringen lassen. Unter Anomalien der Ladung wird das Auftreten eines ,Nachladungsstromes" nach erfolgter "normaler" Ladung und die damit verbundene Rückstandsbildung bezeichnet; unter Anomalien der Leitung die Erscheinung, daB einem konstanten elektrischen Felde kein konstanter Ireitungsstrom des Dielektrikums entspricht, sondern daß dieser eine zeitliche Abnahme zeigt.

Die beiden Phänomene sind insofern zu trennen, als der erstere sich ausgesprochen nur bei festen Dielektriken findet,

1) Vgl. z. B. H. Düggelin, Vierteljahrsschr. d. naturforsch. Ges. Zürich 40. p. 121. 1895. 
der letztere bei flüssigen; es ist jedoch denkbar, daB auch Übergänge und gleichzeitiges Auftreten beider Effekte vorkommen. Der erste wird durch die v. Schweidler-Wagnersche Theorie auf das Vorhandensein dielektrischer Nachwirkung zurückgeführt, der letztere auf Ionenloitung. Die dielektrische Nachwirkung zeigt Proportionalität mit der Feldstärke und „Umkehrbarkeit“, d. h. sie befolgt das „Superpositionsprinzip"; auf Grund dieser Eigenschaften ist eine Vorausberechnung der Wechselstromverluste aus statischen Beobachtungen möglich. Die Leitungserscheinungen sind nicht „umkehrbar"; sie ergeben im Wechselfelde einen Verlust, der genau der verbrauchten Jouleschen Stromwärme entspricht.

Unsere Rechnungen stïtzen sich auf die Kenntnis des Verlaufes der Anomalien bei sehr kleinen Zeiten. Während bei flüssigen Dielektriken bei kleinen Zeiten eine konstante Leitfähigkeit zu finden ist, nimmt der Nachladungsstrom fester Dielektriken mit abnehmender Zeit sehr stark zu, ohne dab das Erreichen eines endlichen Grenzwertes erkennbar ist. Iieser Verlauf läßt sich in gater Näherung darstellen durch die Gleichung:

$$
i=E C_{0} \beta t^{-n} ; \quad 0<n<1
$$

und führt zu einem Fiffektverlust im Wechselfelde, der den Verlust durch stationäre Leitung um mehr als das Hundertfache überwiegen kann. Da dieser Verlust mit der Frequenz zunimmt (in erster Näherung proportional), ergibt sich bei hohen Frequenzen daraus die Folgo, daß Kondensatoren mit dielektrischer Nachwirkung bedeutend höhere Verluste antweisen können als Flüssigkeitskondensatoren, selbst wenn bei niederen Frequenzen diese die größeren Verluste besitzen.

Der Vergleich mit den direkten Messungen zeigt, daß die zum 'Teil genäherte Art unserer Rechnung statthaft ist. Es ergibt sich bei festen Dielektriken ein Effektverlust, der fast vollständig dielektrischer Nachwirkung zugeschrieben werden muB. Neben den ganz geringen Verlusten, die von einem gewissen Leitvermögen herrühren, sind Verluste, die nur in dem Wechseln des Feldes ihren Grund haben, nicht nachzuweisen. Als solehe wären z. B. die Absorption der elektrischen Sehwingungen im Dielektrikum, analog wie sie 
Drud $\left.\theta^{1}\right)$ im Zusammenhange mit den Dispersionserscheinungen bei zahlréichen Flüssigkeiten fand, denkbar. Diese Untersuchungen sind aber bei so kleinen Schwingungsdauern ausgeführt $\left(T \sim 10^{-8}\right.$ Sek.), daß ihre GröBe mit der Schwingungsdauer intramolekularer Vorgänge vergleichbar ist, während bei technischen W'echselströmen dies nicht der Fall ist. Es sind also die von uns gemessenen Verluste ganz anderer Natur; sie scheinen insbesondere vom Aggregatzustand abhängig zu sein. Solange sie ihrem Wesen nach vicht näher bekannt sind, wird es berechtigt sein, von "dielektrischer Nachwirkung" oder von ,viskoser Hysterese" zu sprechen, ohne mit diesen Ausdrücken ein spezielles molekularphysikalisches Bild zu verbinden. Für uns mag es genügen, den Zusammenhang zwischen den statischen Erscheinungen und den Effektverlustion in elektrischen Wechselfeld an einigen Beispielen quantitativ nachgewiesen und so zur Stützung der v. Schweidler. Wagnerschen Theorie beigetragen zu haben. Letzterer selbst muß man trotz einer gewissen Anschaulichkeit hauptsächlich formalen Charakter zuschreiben; es ist aber bemerkenswert, daß sie, auf einfacher Grundlage aufgebaut, sehr umfassend ist. Inwiefern sie sich auf das Verhalten flüssiger Dielektriken übertragen läßt, für welches einige Analogien vorliegen, und inwiefern die große Ähnlichkeit mit den Erscheinungen der elastischen Nachwirkung einem inneren $\mathrm{Zu}-$ sammenhang entspricht, sind Fragen, deren Beantwortung für die Lösung des gesamten Problems von größter Bedeutung wäre, die aber auch auf große Schwierigkeiten führen dürften.

\section{Zusammenfassung.}

Es wurde an festen und flüssigen Dielektriken der Zusammenhang zwischen den Effektverlusten im elektrischen Wechselfeld und den anomalen Erscheinungen der Ladung (Nachladung, Rückstandsbildung) und der Leitung (Abweichungen vom $\mathrm{Ohmschen} \mathrm{(Gesetz)} \mathrm{untersucht.}$

1. Es wurden Methoden ausgearbeitet:

a) Mit dem Helmholtzschen Pendel zur Bestimmung des Stromverlaufes in Kondensatoren unter Einfluß einer

1) P. Drude, Wied. Ann. 61. p. 466. 1897; 59. p. 17. 1896; 58. p. 1. 1896; 60. p. 500 . 1897 . 
konstanten Spannung zu Zeiten, die der Periodendauer technischer Wechselströme vergleichbar sind.

b) Mit dem Elektrodynamometer ron Sumpner in Resonanzschaltung zur Bestimmung der Effehtverluste von Kondensatoren bei-nicht zu hohen Spannungen und Frequenzen.

2. Es ergab sich:

a) Die Wechselstromverluste fester Dielektriken rühren ïberwiegend von dielektrischer Nachwirkung her und können aus zwei, durch statische Messungen gewonnene Materialkonstanten berechnet werden. Die Verluste durch Joulesche Wärme betragen geringe Bruchteile $(<1$ Proz.) der Gesamtverluste. Zusätzliche Verluste anderér Art im Wechselfeld sind nicht nachzuweisen.

b) Die Wechselstromverluste flüssiger Dielektriken sind auf Leitung durch Ionen zurückzuführen und verschwinden mit zunehmender Reinheit der Substanzen.

Die Resultate enthalten eine Bestätigung der v. Schweidler-Wagnerschen Theorie der unvollkommenen Dielektriken.

Die vorliegende Arbeit wurde vom Sommer 1913 bis Frühjahr 1915 mit Unterbrechung eines halben Jahres im Physikalischen Institut der Universität Zürich ausgeführt. Meinem verehrten Chef, Herrn Prof. Dr. A. Kleiner, der mich anf das Untersuchungsgebiet hinwies, möchte ich für das große Entgegenkommen, das er mir stets bewies, vielen Dank aussprechen.

Zürich, 13. Juni 1915.

(Eingegangen 7. August 1915.) 